\title{
DEFORMATION THEORY AND LIMITING MIXED HODGE STRUCTURES
}

\author{
MARK GREEN AND PHILLIP GRIFFITHS
}

\section{OutLine}

\author{
I. Introduction \\ II. Deformation theory \\ III. Proof of Theorems I and I' \\ IV. Proof of Theorem II \\ V. Proof of Theorem III \\ VI. The hierarchy of mixed Hodge structures \\ References
}

\section{INTRODUCTION}

This paper was motivated by the following question: Recall that for a smooth projective variety $X$ whose polarized Hodge structure on $H^{n}(X, \mathbb{Q})_{\text {prim }}$ leads to a period point $\Phi(X) \in D$, the period domain for polarized Hodge structures of a fixed type, the differential

$$
\Phi_{*}: T_{X} \operatorname{Def}(X) \rightarrow T_{\Phi(X)} D
$$

from the tangent space to the Kuranishi space $\operatorname{Def}(X)$ to the tangent space $T_{\Phi(X)} D$ is expressed cohomologically by the inclusion $T_{X} \operatorname{Def}(X) \hookrightarrow \mathbb{E x t}_{\mathcal{O}_{X}}^{1}\left(\Omega_{X}^{1}, \mathcal{O}_{X}\right) \cong$ $H^{1}\left(T_{X}\right)$, and the resulting natural maps on the associated graded to $F^{\bullet} \mathbb{H}^{m}\left(\Omega_{X}^{\bullet}\right)$ induced from

$$
\operatorname{Ext}_{\mathcal{O}_{X}}^{1}\left(\Omega_{X}^{1}, \mathcal{O}_{X}\right) \otimes \mathbb{H}^{m}\left(\Omega_{X}^{\bullet}\right) \rightarrow \mathbb{H}^{m}\left(\Omega_{X}^{\bullet}\right) \mathbb{1}
$$

Our question was how to extend this formalism to the case where $X$ is singular, having singularities of the type that arise by semi-stable reduction in a family of projective varieties $x \stackrel{f}{\rightarrow} S$ whose general member is smooth. This question was studied by Friedman [Fr1] when $\operatorname{dim} S=1$. We were interested in amplifying and extending his results, emphasizing the development of a formalism that lends itself to the computation of examples. In the course of trying to carry this out we have found that there is an interesting story surrounding the relationships among the various mixed Hodge structures associated to $X$ and its $1^{\text {st }}$ order neighborhood in $X$, and one of the purposes of this paper has turned out to be to amplify and clarify these relationships in the context of deformation theory. Here for us the works Fr2], St1 and St2 have been very important when $\operatorname{dim} S=1$, as has the extension of [St1] to a general $S$ by Fujisawa [Fu1, Fu2. In fact, this is a partly

\footnotetext{
${ }^{1}$ In more classical notation $\Theta_{X}=T_{X}$, this is the map$$
H^{1}\left(\Theta_{X}\right) \otimes H^{m-p}\left(\Omega_{X}^{p}\right) \rightarrow H^{m-p+1}\left(\Omega_{X}^{p-1}\right)
$$$$
\text { induced from the contraction } \Theta_{X} \otimes \Omega_{X}^{p} \rightarrow \Omega_{X}^{p-1} \text {. }
$$ 
expository paper, drawn from, reinterpreting and building on the works [Fr1, Fr2, [St1, [Zu, PS, [St2, [Fu1, Fu2, CKS1, [KP2, GGR] and others.

To address the question stated above one is led to focus on the singular variety $X$ and its first order deformations. For the case when $X$ is a normal crossing variety this is done in $\mathrm{Fr} 2$. Here motivated by the semi-stable reduction theorem in $\mathrm{AK}$ we shall assume more generally that

\section{$X$ is locally a product of normal crossing varieties.}

This means locally in $\mathbb{C}^{n}$ with coordinates $x_{1}, \ldots, x_{n}$ we have a sequence $1 \leqq i_{1}<$ $i_{2}<\cdots<i_{k} \leqq n$ with index blocks $I_{1}=\left\{1, \ldots, i_{1}\right\}, I_{2}=\left\{i_{1}+1, \ldots, i_{2}\right\}, \ldots$, and then $X$ is locally given by

$$
x_{I_{1}}=0, \ldots, x_{I_{k}}=0
$$

where $x_{I_{1}}=x_{1} \cdots x_{i_{1}}, x_{I_{2}}=x_{i_{1}+1} \cdots x_{i_{2}}, \ldots$ The usual locally normal crossing variety is the case $k=12$ The deformation theory of such varieties is well understood $[\mathrm{Pa}$, and for simplicity of exposition in this paper we shall abuse notation and set

$$
T_{X} \operatorname{Def}(X)=\mathbb{E x t}_{\mathcal{O}_{X}}^{1}\left(\Omega_{X}^{1}, \mathcal{O}_{X}\right) .
$$

The abuse of notation is because here the right-hand side is the space of deformations of $X$ over $\Delta_{\epsilon}=: \operatorname{Spec} \mathbb{C}[\epsilon], \epsilon^{2}=0$, so that it is only the Zariski tangent space to the Kuranishi space $\operatorname{Def}(X)$. In general there may be obstructions to lifting deformations defined over the Zariski tangent space, but this issue will play no role in what follows ${ }^{3}$ In fact, one of the main points is that the theory of limiting mixed Hodge structures for 1-parameter families depends only on the $1^{\text {st }}$ order neighborhood of the singular variety, a point that is implicit in Fr2] and explicit in a somewhat different form in St2.

We shall make the crucial assumption that there exists a $\xi \in \mathbb{E x t}_{\mathcal{O}_{X}}^{1}\left(\Omega_{X}^{1}, \mathcal{O}_{X}\right)$ such that for every $x \in X$ the image $\xi_{x}$ of $\xi$ in the natural map

$$
\operatorname{Ext}_{\mathcal{O}_{X}}^{1}\left(\Omega_{X}^{1}, \mathcal{O}_{X}\right) \rightarrow \operatorname{Ext}_{\mathcal{O}_{X}}^{1}\left(\Omega_{X}^{1}, \mathcal{O}_{X}\right)_{x}
$$

smooths to $1^{\text {st }}$ order the singularity at $x$. Equivalently, for every $x \in X$ the global deformations of $X$ over $\Delta_{\epsilon}$ map to smoothing deformations of the germ $X_{x}$ of $X$ at $x$. The smoothing deformations of (1.2) are given by $x_{I_{j}}=t_{j}$ and they have tangents $\sum_{i=1} \lambda_{i} \partial_{t_{i}}$ where all $\lambda_{i} \neq 0$. We denote by

$$
T_{X}^{0} \operatorname{Def}(X) \subset T_{X} \operatorname{Def}(X)
$$

the open set of all $\xi \in T_{X} \operatorname{Def}(X)$ whose localizations are smoothing deformations of $X_{x}$ for every $x \in X$.

\footnotetext{
${ }^{2}$ There is an important distinction between the case when $X$ is locally a normal crossing variety and when it is globally such. By a combination of blowings up and base changes the former may be reduced to the later, and for both theoretical and notational purposes this is generally done. For computational purposes the former is frequently more convenient; e.g., for irreducible nodal curves. In this paper we shall restrict to the global normal crossing case and its analogue when $X$ is locally a product of normal crossing varieties. However, we expect that the discussion given below will remain valid in the more general case, and some of our examples are carried out in the local normal crossing case. The formalism in De and St2 allows one to handle the general theory when $X$ is locally a normal crossing variety.

${ }^{3}$ One may make the blanket assumption that all $1^{\text {st }}$ order deformations are unobstructed, and then at the end note that this assumption has never been used.
} 
We define the pair $(X, \xi)$ to be projective in case there is a very ample line bundle $L \rightarrow X$ such that $L$ extends to $X_{\xi}$. This can be expressed cohomologically in a standard way, and we shall assume it to always be the case.

A limiting mixed Hodge structure $\left(V, W_{\bullet}, F^{\bullet}\right)$ is given by a $\mathbb{Q}$-vector space $V$, a weight filtration $W_{\bullet}$ and Hodge filtration $F^{\bullet}$ that define a mixed Hodge structure, and where there exists a nilpotent $N \in \operatorname{End}(V)$ such that (i) $W_{\bullet}=W_{\bullet}(N)$ is the monodromy weight filtration, and (ii) for the integer $m$ around which the monodromy weight filtration is centered, the $N^{k}: \mathrm{Gr}_{m+k}^{W \cdot} V \stackrel{\sim}{\rightarrow} \operatorname{Gr}_{m-k}^{W \cdot} V$ are isomorphisms for $0 \leqq k \leqq m$. The limiting mixed Hodge structure is polarizable if there exists a $Q: V_{\otimes} V \rightarrow \mathbb{Q}$ with $Q(u, v)=(-1)^{m} Q(v, u)$, and an $N \in \operatorname{End}_{Q}(V)$ as above such that the primitive spaces ker $N^{k+1}$ above are polarized Hodge structures via $Q_{k}(u, v)= \pm Q\left(N^{k} u, v\right)$ (cf. CKS1). Two limiting mixed Hodge structures $\left(V, W_{\bullet}, F^{\bullet}\right)$ and $\left(V, W_{\bullet}^{\prime}, F^{\prime \bullet}\right)$ are equivalent if $W_{\bullet}^{\prime}=W_{\bullet}$, and if ${F^{\prime}}^{\bullet}=\exp (z N) F^{\bullet}$ for some $z \in \mathbb{C}$. We will denote by $\left[V, W_{\bullet}, F^{\bullet}\right]$ an equivalence class of limiting mixed Hodge structures.

We shall use the term standard family to mean that $x_{\Delta} \rightarrow \Delta$ is a projective mapping where $X_{\Delta}$ is smooth, the fibres $X_{t}=\pi^{-1}(t)$ are smooth for $t \neq 0$, and $X_{0}=X$ is a reduced normal crossing variety.

Theorem I: Canonically associated to each $\xi \in T_{X}^{0} \operatorname{Def}(X)$ is a limiting mixed Hodge structure $\left(V_{\xi}, W_{\bullet}, F_{\xi}^{\bullet}\right)$. In case $X$ is a normal crossing variety and $\xi$ is tangent to an arc $\Delta \subset \operatorname{Def}(X)$ giving a standard family $X_{\Delta} \stackrel{\pi}{\rightarrow} \Delta$ with $\pi^{-1}(0)=$ $X$, this limiting mixed Hodge structure is the one associated to the family and $\xi \in T_{0}(\Delta)$.

This result is largely an amalgam and slight extension of those in [Fr2] and [St2]. A key point is to note that the data $(X, \xi)$ gives a standard family $X_{\xi} \rightarrow \Delta_{\epsilon}$, together with an extension

$$
0 \rightarrow \mathcal{O}_{X} \rightarrow \Omega_{X_{\xi}}^{1} \otimes \mathcal{O}_{X} \rightarrow \Omega_{X}^{1} \rightarrow 0
$$

of $\mathcal{O}_{X}$-modules 4 A second key point is to show that, as explained in section III below, (I.4) gives an exact sequence

$$
0 \rightarrow \mathcal{O}_{X} \rightarrow \Omega_{X_{\xi}}^{1}(\log X) \otimes \mathcal{O}_{X} \rightarrow \Omega_{X_{\xi} / \Delta_{\epsilon}}^{1}(\log X) \otimes \mathcal{O}_{X} \rightarrow 0
$$

where in the case of a standard family $x_{\Delta} \rightarrow \Delta$ restricting to $x_{\xi} \rightarrow \Delta_{\epsilon}$

$$
\Omega_{X_{\xi} / \Delta_{\epsilon}}^{1}(\log X) \otimes \mathcal{O}_{X}=\Omega_{X_{\Delta / \Delta}}^{1}(\log X) \otimes \mathcal{O}_{X} .
$$

The vector space in the limiting mixed Hodge structure is

$$
V_{\xi}=\mathbb{H}^{m}\left(\Omega_{\mathfrak{X}_{\xi} / \Delta_{\epsilon}}(\log X) \otimes \mathcal{O}_{X}\right)
$$

and $F_{\xi}^{\bullet}$ is induced from the "bête" filtration on $\Omega_{X_{\xi} / \Delta_{\epsilon}}(\log X) \otimes \mathcal{O}_{X}$. The monodromy logarithm is induced from the connecting homomorphisms arising from (I.5). The $\mathbb{Q}$-structure and properties of the monodromy logarithm and resulting monodromy weight filtration are more subtle to define and treat (cf. [St1], $\mathrm{Zu}$ ] and chapter 11 in $[\mathrm{PS}]$ ).

\footnotetext{
${ }^{4}$ We are here extending the notion of a standard family to include the smooth non-reduced scheme $X_{\xi}$ with structure sheaf $\mathcal{O}_{X_{\xi}}$ locally isomorphic to $\mathcal{O}_{X}[\epsilon]$. We will also say that fibres over $\Delta_{e}^{*}$ are smooth.
} 
We note that the usual ambiguity in either the Hodge filtration or the $\mathbb{Q}$-structure in the limiting mixed Hodge structure associated to $X \rightarrow \Delta$, ambiguity that depends on a choice of parameter $t$, is removed by considering the data $(X, \xi)$.

A subtle point, one that will be further explained below, is this: For $X$ a smoothable normal crossing variety the singular locus $D$ will have connected components $D_{a}$. Then we will see that $\operatorname{Ext}_{\mathcal{O}_{X}}^{1}\left(\Omega_{X}^{1}, \mathcal{O}_{X}\right) \cong \oplus \mathcal{O}_{D_{a}}$, and in the basic exact sequence (II.1) a global $1^{\text {st }}$ order deformation $\xi \in \mathbb{E x t}_{\mathcal{O}_{X}}^{1}\left(\Omega_{X}^{1}, \mathcal{O}_{X}\right)$ will induce $\xi_{D_{a}} \in H^{0}\left(\mathcal{O}_{D_{a}}\right)$. The condition that $\xi$ be to $1^{\text {st }}$ order smoothing along $D_{a}$ is that $\xi_{D_{a}} \neq 0$. Then the equivalence class of the limiting mixed Hodge structure in Theorem $\square$ depends only on the $\xi_{D_{a}}$ and not on the global $\xi$ that maps to the $\xi_{D_{a}}$ 's. In fact, given a collection of non-zero $\xi_{D_{a}}$ 's, we may construct a limiting mixed Hodge structure provided that there is a global smoothing $\xi$; the particular $\xi$ does not matter 5

To handle several variable families we shall consider a vector

$$
\xi \in \mathbb{E x t}_{\mathcal{O}_{X}}^{1}\left(\Omega_{X}^{1}, \mathcal{O}_{X}^{\ell}\right)=\stackrel{\ell}{\oplus} T_{X} \operatorname{Def}(X)
$$

with the property that for $\lambda=\left(\lambda_{1}, \ldots, \lambda_{\ell}\right)$ and

$$
\boldsymbol{\xi}_{\lambda}=\lambda_{1} \xi_{1}+\cdots+\lambda_{\ell} \xi_{\ell}, \quad \lambda_{i} \neq 0
$$

we have

$$
\xi_{\lambda} \in T_{X}^{0} \operatorname{Def}(X) .
$$

For $\Delta_{\epsilon}=\Delta_{\epsilon_{1}} \times \cdots \times \Delta_{\epsilon_{\ell}}$, it will be seen that we then have a family

$$
x_{\xi_{\lambda}} \rightarrow \Delta_{\epsilon}
$$

with smooth fibres over $\Delta_{\epsilon}^{*}=\Delta_{\epsilon_{1}}^{*} \times \cdots \times \Delta_{\epsilon_{\ell}}^{*}$. We think of this as a space of $1^{\text {st }}$ order deformations that deform $X$ to a "less singular" variety along the axes but which smooth $X$ when we deform into the interior 6 There is then a several variables analogue of Theorem $\amalg$ where the terms in the statement will be explained in the text.

TheOREM I': Associated to $\boldsymbol{\xi} \in \mathbb{E x t}_{\mathcal{O}_{X}}^{1}\left(\Omega_{X}^{1}, \mathcal{O}_{X}^{\ell}\right)$ satisfying the condition that (I.6) holds, there is a several variable limiting mixed Hodge structure $\left(V_{\boldsymbol{\xi}}, W_{\bullet}, F_{\boldsymbol{\epsilon}}^{\bullet}\right)$ in the sense of [CKS1. In case $X$ is a normal crossing variety and $\boldsymbol{\xi}_{\lambda}$ is tangent to an arc $\Delta_{\lambda} \subset \operatorname{Def}(X)$, this limiting mixed Hodge structure is the one associated to the standard family $X_{\Delta_{\lambda}} \stackrel{\pi}{\rightarrow} \Delta_{\lambda}$ with $\pi^{-1}(0)=X$.

As will be discussed below, for the last statement in the theorem the general case when $X$ is locally of the form (I.3) seems to be open (cf. [Fu1], Fu2]), and we will discuss a geometric reason for this.

\footnotetext{
${ }^{5}$ As we hope to discuss further in a work in progress, this is related to the theorem of CattaniKaplan [CK] that the weight filtration $W \bullet(N)$ is independent of $N$ in the interior of a monodromy cone, and the result in CKS1 that the equivalence class of the limiting mixed Hodge structure is independent of the direction of approach from the interior of the cone.

${ }^{6}$ In the paper $\mathrm{KN}$ the definition of a normal crossing variety with logarithmic structure is introduced. The presence of a logarithmic structure is equivalent to $d$-semi-stability in the sense of [Fr2 (cf. [I.6 below). A deformation theory for normal crossing varieties with logarithmic structure is then introduced. In the context of this paper this theory amounts to deformations of $X$ that independently smooth the connected components of the singular locus $D$ of $X$, modulo equisingular deformations. The log-geometry formalism nicely lends itself to computation of examples for Calabi-Yau varieties.
} 
Detailed proofs of Theorems I and I', especially for the latter, will not be given below. The argument for Theorem 1 consists largely of proof analysis of those in the references [Fr2], St1 and [St2] and will be addressed more fully in a work in progress. For Theorem II the construction of a mixed Hodge structure follows largely from our construction given below and Fu1, Fu2. The construction of a limiting mixed Hodge structure requires more work and will be taken up in the work in progress. We will however try to point out some of the key points in both of these arguments.

For the analogue of (I.1) we have

Theorem II: The class $\xi \in \mathbb{E x t}_{\mathcal{O}_{X}}^{1}\left(\Omega_{X}^{1}, \mathcal{O}_{X}\right)$ in (I.4) defines a natural class $\xi^{(1)} \in$ $\mathbb{E x t}_{\mathcal{O}_{X}}^{1}\left(\Omega_{\mathcal{X}_{\varepsilon} / \Delta_{\epsilon}}^{1}(\log X) \otimes \mathcal{O}_{X}, \mathcal{O}_{X}\right)$ corresponding to (1.5), and the $1^{\text {st }}$ order variation of the limiting mixed Hodge structure is expressed as the natural mapping

$$
\begin{aligned}
\operatorname{Ext}_{\mathcal{O}_{X}}^{1}\left(\Omega_{X_{\xi} / \Delta_{\epsilon}}^{1}(\log X) \otimes \mathcal{O}_{X}, \mathcal{O}_{X}\right) & \\
& \left.\rightarrow \operatorname{End}_{\text {LMHS }} \mathbb{H}^{m}\left(\Omega_{X_{\xi} / \Delta_{\epsilon}}^{\bullet}(\log X) \otimes \mathcal{O}_{X}\right)\right]
\end{aligned}
$$

Here, $\mathbb{H}^{m}\left(\Omega_{\dot{X}_{\xi} / \Delta_{\epsilon}}^{\bullet}(\log X) \otimes \mathcal{O}_{X}\right)=V_{\xi}$ is the vector space underlying the limiting mixed Hodge structure in Theorem \ and End $V_{\xi}$ that preserve the structure as a limiting mixed Hodge structure as explained below.

Again the terms in the statement will be explained in the text. An informal way to think about this result is this: Denoting by $\operatorname{Def}(X, \xi)$ the deformations of the pair $(X, \xi)$, we have a natural extended period mapping

$$
\operatorname{Def}(X, \xi) \rightarrow \check{D}
$$

that assigns to $\xi \in T_{X}^{0} \operatorname{Def}(X)$ the well-defined point $F_{\xi}^{\bullet} \in \check{D}$, the dual space to the period domain $D$ consisting of filtrations of $V_{\xi}$ that satisfy only the $1^{\text {st }}$ HodgeRiemann bilinear relation. Then in the map in Theorem II might be thought to be the differential

$$
T_{(X, \xi)} \operatorname{Def}(X, \xi) \rightarrow T_{F_{\xi}} \check{D}
$$

of the extended period mapping. This is not the case, as will be made precise in Section IV below. The issue is more subtle in that $\xi$ gives not only a well-defined limiting mixed Hodge structure, not just an equivalence class of such, but also defines a $1^{\text {st }}$ order variation of that limiting mixed Hodge structure. This is the information in $\xi^{(1)}$. At first glance one might think that since it takes the tangent vector $\xi$ to define $F_{\xi}^{\bullet}$, the information in $\xi^{(1)}$ which gives the variation of the entire limiting mixed Hodge structure would be of $2^{\text {nd }}$ order. But this is not correct, and

\footnotetext{
${ }^{7}$ The notation $\xi^{(1)}$ has been used because the construction of the sequence (I.5) from I.4 resembles that of the construction of the first prolongation in the theory of exterior differential systems. The group $\mathbb{E x t}_{\mathcal{O}_{X}}^{1}\left(\Omega_{x_{\xi} / \Delta_{\epsilon}}^{1}(\log X) \otimes \mathcal{O}_{X}, \mathcal{O}_{X}\right)$, which may be defined if there exists a logarithmic structure on $X$, appears naturally in the deformation theory of smooth logarithmic varieties (cf. $\left.\mathrm{A}^{\dagger}\right)$.

Referring to footnote 17 below, in the setting of log-analytic geometry the important monograph $[\mathrm{KU}]$ contains a treatment of the differential of the period map at infinity for standard families $x_{\Delta} \rightarrow \Delta$ (cf. Theorem 4.4.8). In case the $\xi$ in Theorem II arises as the tangent vectors at the origin we believe that those results should be equivalent.
} 
it was in trying to understand this loint that we were led to most of the other topics in this paper.

We will however see by example that $\xi^{(1)}$ contains strictly more information than the differential at the origin of the Kato-Usui map $\underline{\mathrm{KU}}$

$$
\Delta \rightarrow \Gamma_{T} \backslash D_{N} .
$$

Here $D_{N}=D \cup B(N)$ is the period domain $D$ with the boundary component $B(N)$ attached to $D$, where $B(N)$ consists of all equivalence classes of limiting mixed Hodge structures with monodromy logarithm $N$ and where $\Gamma_{T}=\left\{T^{\mathbb{Z}}\right\}$ with $T=\exp N$ is the local monodromy group (cf. Section IV below for an explanation of the notations and terms used). It is in this sense that Theorem II provides an answer to our original question. The term "expressed" means that in examples $\mathbb{E x t}_{\mathcal{O}_{X}}^{1}\left(\Omega_{X_{\xi} / \Delta_{\epsilon}}^{1}(\log X) \otimes \mathcal{O}_{X}, \mathcal{O}_{X}\right)$ will have algebro-geometric meaning and the pairing is a cup-product. We will see by example that the additional information is non-trivial and somewhat subtle 8

As will be explained in Section V below, associated to a polarized limiting mixed Hodge structure is a reduced limit period mapping and distinguished point

$$
F_{\infty}^{\bullet} \in \partial D
$$

where $D=G_{\mathbb{R}} / H$ is a period domain ( $\mathrm{KP} 1$, KP2, GGK, GG] and GGR]). The boundary $\partial D$ is stratified into finitely many $G_{\mathbb{R}}$-orbits and their geometry is a much studied and very interesting topic (GGK, [FHW]).

On the other hand, the vector space $T_{X} \operatorname{Def}(X)$ is stratified by open sets $T_{X}^{0} \operatorname{Def}(X)_{I}$ contained in linear subspaces $T_{X} \operatorname{Def}(X)_{I} \subset T_{X} \operatorname{Def}(X)$. In the text we will explain this in case $X$ is a normal crossing divisor, which is the only case for which thus far we have a result. Then the strata correspond to subsets of the set of connected components of the singular locus $X_{\text {sing }}$ of $X$. The subspace $T_{X}^{0} \operatorname{Def}(X)$ is the open stratum of smoothing deformations; the other strata correspond to the components that are smoothed when $X$ deforms in the directions of that strata. The opposite extreme to $T_{X}^{0} \operatorname{Def}(X)$ is the linear subspace $T_{X} \operatorname{Def}^{\mathrm{es}}(X) \cong H^{1}\left(\operatorname{Ext}_{\mathcal{O}_{X}}^{0}\left(\Omega_{X}^{1}, \mathcal{O}_{X}\right)\right)$ of equisingular deformations. It seems reasonable to expect, but we are not aware of a proof in the literature, that $\xi \in T_{X}^{0} \operatorname{Def}(X)_{I}$ corresponds to the limit in a variation of mixed Hodge structures over the punctured disc ([St-Zu $)$.

Leaving this important issue aside, we return to the deformation theory and limiting mixed Hodge structures in the several parameter case. In the study of limiting mixed Hodge structures over higher dimensional base spaces (CKS1) there are a number of cone structures that enter:

(i) the stratification of abelian subspaces $\mathfrak{A} \subset \mathfrak{g}^{\text {nilp }}$ induced by the $G$-orbit structure on $\mathfrak{g}^{\text {nilp }}$ ([R0] and references cited therein);

(ii) the stratification of nilpotent cones as in [CKS1] and [KU] (cf. AMRT] for the classical weight one case);

\footnotetext{
${ }^{8}$ In very classical terms one may write the period matrix $\Omega(t)$ in block form where the blocks $\Omega_{i}(t)$ are polynomials in $\log t$ with holomorphic coefficients and where the remaining blocks $\Omega_{\alpha}(t)$ are holomorphic at $t=0$. The differential of the map to $\Gamma_{T} \backslash D_{N}$ records the derivatives $\Omega_{\alpha}^{\prime}(0)$ of the holomorphic terms, while (I.7) has the effect of regularizing the logarithmically divergent integrals that give the $\Omega_{i}(t)$ and then taking the linear part $\Omega_{i}^{\prime}(0)$ at $t=0$ of that regularization. The $\Omega_{\alpha}^{\prime}(0)$ and $\Omega_{i}^{\prime}(0)$ record the variation in the full extension data in the limiting mixed Hodge structure.
} 
(iii) the stratification of $\partial D$ by $G_{\mathbb{R}}$-orbits and its relation to reduced limit period mappings [KP1], KP2], GG], GGR] and [Ro] and work in progress by Kerr, Pearlstein and Robles; and

(iv) the stratification of $T_{X} \operatorname{Def}(X)$, as explained below for $X$ a normal crossing divisor, and which we feel can reasonably be expected to extend to the case where $X$ is locally a product of normal crossing divisors.

The basic known result, due to Robles $[\mathrm{Ro}$, is that the interiors of the strata in (ii) map to strata in (i), and as a consequence to strata in (iii) 9 Her argument makes full use of the deep properties of several variable nilpotent orbits CKS1 and of the classification of $G_{\mathbb{R}}$-orbits in $\mathfrak{g}_{\mathbb{R}}^{\text {nilp }}$ (cf. the references in [Ro]). An algebro-geometric version of Robles' result might be that at the tangent space level strata in (iv) map to strata in (iii). The theorem to be described now is a partial result in this direction.

In the setting of the Cattani-Kaplan-Schmid theory there are defined nilpotent cones

$$
\sigma=\operatorname{span}_{\mathbb{Q}>0}\left\{N_{1}, \ldots, N_{\ell}\right\}
$$

where the $N_{i} \in \mathfrak{g}^{\text {nilp }}$ are linearly independent commuting nilpotent transformations and several variable nilpotent orbits $\left(F^{\bullet}, \sigma\right)$. Here, $F^{\bullet} \in \check{D}$ and the conditions

- $\exp \left(z_{1} N_{1}+\cdots+z_{\ell} N_{\ell}\right) \cdot F^{\bullet} \in D$ for all $\operatorname{Im} z_{i} \gg 0$;

- $\left[N_{i}, F^{p}\right] \subset F^{p-1}$

are satisfied. We denote by $\widetilde{B}(\sigma) \subset \check{D}$ the set of several variable nilpotent orbits, and by $B(\sigma)$ the equivalence classes of those orbits under reparametrization $z_{i} \rightarrow$ $z_{i}+\lambda_{i}$. In [KP1], GGK] and GG there are defined reduced limit period mappings for 1-dimensional cones, and the construction can be extended KP2 to the general case to give the reduced limit period map

$$
\Phi_{\infty}: B(\sigma) \rightarrow \partial D
$$

THEOREM III: Let $X$ be a normal crossing variety for which there exists a $\xi \in$ $T_{X} \operatorname{Def}(X)$ that is nowhere vanishing along each component of $X_{\text {sing. }}$. Then there exists a nilpotent cone

$$
\sigma_{X} \subset T_{X} \operatorname{Def}(X) / T_{X}^{\mathrm{es}} \operatorname{Def}(X)
$$

and a several variable limiting mixed Hodge structure in the sense of [CKS1] with the property that under the reduced limit period mapping (I.8) $\widetilde{B}\left(\sigma_{X}\right)$ maps to a $G_{\mathbb{R}}$-orbit in $\partial D$.

This theorem follows from the construction of $\sigma_{X}$ and the result of Robles mentioned above. As mentioned before, it is of interest to see if the construction of $\sigma_{X}$ and the result can be extended to the faces of the cone $\sigma_{X}$.

For our next result we note that given a standard family $x \rightarrow \Delta$ there are the following four types of mixed Hodge structures that may be defined:

(i) the part of the mixed Hodge structure on $H^{*}(X)$ that comes from the limiting mixed Hodge structure 10

(ii) that part of the limiting mixed Hodge structure that may be defined in terms of $X$ alone;

\footnotetext{
${ }^{9}$ For the interior of the full nilpotent cone this result follows from CKS1.

${ }^{10}$ This is ker $N$.
} 
(iii) the limiting mixed Hodge, modulo reparametrizations $F_{\lim }^{\bullet} \sim \exp (z N)$. $F_{\text {lim }}^{\bullet}$ resulting from a change in parameter in the disc, associated to $\mathcal{X} \rightarrow$ $\Delta, 11$ and

(iv) the limiting mixed Hodge structure associated to $(X, \xi)$, where $\xi \in T_{X}^{0} \operatorname{Def}(X)$ is the first order variation of $X$ in $X$.

THEOREM IV: There are strict implications

$$
\text { (iv) } \Longrightarrow \text { (iii) } \Longrightarrow \text { (ii) } \Longrightarrow \text { (i). }
$$

The term "strict implication" means that there is successively more information in (i), (ii), (iii), (iv); the precise meaning of this will be explained in the proof.

We will see that given an abstract $X$ that is locally a normal crossing divisor, the condition that we may construct the data given in (ii) is that there exists a $\xi \in T_{X}^{0} \operatorname{Def}(X)$ that is smoothing to $1^{\text {st }}$ order; the actual data will not depend on the particular $\xi$ but rather will depend on the $\xi_{D_{a}}$ 's as discussed above. A limiting mixed Hodge structure will decompose into $N$-strings under the action of the monodromy logarithm $N$. This decomposition may be pictured as

$$
\begin{gathered}
H^{0}(-m) \longrightarrow \\
H^{1}(-m+1) \longrightarrow \\
\cdots \longrightarrow H^{1}(-1) \longrightarrow H^{0}(-1) \longrightarrow H^{0} \\
\vdots \\
H^{m}
\end{gathered}
$$

where $H^{k}$ is a pure Hodge structure of weight $k 12$ We may think of (I.9) as giving the primitive decomposition in the associated graded to a limiting mixed Hodge structure, together with the iterated action of $N$ on the primitive spaces. Then our result pertaining to (ii) is

Theorem V: The terms $H^{m-j}(-i), 0 \leqq i \leqq m-j$ in (1.9), together with the $N$-maps between them, may be constructed from $X$ alone.

We will also see for $[\xi] \in \mathbb{P} T_{X}^{0} \operatorname{Def}(X)$ with localizations $\xi_{D_{a}}$ along the components of $D_{a}$ of $X_{\text {sing, }}$, we will have

$$
\text { (iii) } \longleftrightarrow\left(X,\left[\xi_{D_{a}}\right]^{\prime} \mathrm{s}\right),
$$

and where the brackets refer to the corresponding point in the designated projective space and the symbol "«" means that the data on each side are equivalent.

We hope that this result will clarify exactly what input is needed to be able to define the limiting mixed Hodge structures, or the parts thereof, that are associated to a degeneration $X \rightarrow \Delta$ of a smooth projective variety 13 All of (i)-(iv) require knowledge of at most the $1^{\text {st }}$ order neighborhood of $X$ in $\mathcal{X}$. It is worth noting that even though the central fibre $X$ is in general not uniquely definable 14 the ambiguity

\footnotetext{
${ }^{11}$ This is by definition the same as an equivalence class of limiting mixed Hodge structures.

${ }^{12}$ If one thinks of $N$ as being completed to an $\mathrm{sl}_{2}$-triple, then the $N$-strings are composed from the irreducible pieces in the decomposition of the $\mathrm{sl}_{2}$-module. The $H^{k}$ 's on the right end may themselves be Tate twists of lower weight Hodge structures.

${ }^{13}$ Its proof mainly consists of "proof analysis" of the construction of the limiting mixed Hodge structure in [St1], $\mathrm{Zu}$ ] and [St2]. Our main new point is to focus from the outset on the pair $(X, \xi)$.

${ }^{14}$ Exceptions include stable curves, principally polarized abelian varieties and marked $K 3$ surfaces, all of which have "good" global moduli spaces.
} 
in the limiting mixed Hodge structures "washes out" in the constructions (ii), (iii), (iv) 15

We note that the traditional approach in the study of the behavior of the polarized Hodge structures in a degenerating family of smooth projective varieties is to start with a family $X^{*} \rightarrow \Delta^{*}$ with unipotent monodromy $T$. To this we may either associate a period mapping

$$
\Phi: \Delta^{*} \rightarrow \Gamma_{T} \backslash D, \quad \Gamma_{T}=T^{\mathbb{Z}},
$$

and then by $[\mathrm{Sc}$ to this period mapping associate an equivalence class of limiting mixed Hodge structures. Or more algebro-geometrically we may complete $X^{*} \rightarrow \Delta^{*}$ to a standard family to which by [St1] we may associate the same equivalence class of limiting mixed Hodge structures 16 In this paper we are starting with the central fibre $X$ with only the assumptions that (a) $X$ is projective and is locally a normal crossing divisor, or more generally that it is locally a product of normal crossing divisors, and (b) there exists a $\xi \in T_{X} \operatorname{Def}(X)$ that is to $1^{\text {st }}$ order smoothing and preserves the ample line bundle. We hope that this helps to explain the title of this work.

In what follows we shall use $X$ to denote both a compact analytic variety and a germ of an analytic variety; we hope the context will make clear to which we are referring. When $X$ is a compact analytic variety and $x \in X$ we shall denote by $X_{x}$ the germ of analytic variety defined by localizing $X$ at $x$.

The other notations we have used are either standard or will be noted where introduced. For our variety $X$ we will have $\operatorname{dim} X=n$, and we shall generally consider cohomology and hypercohomology in degree $m$ (e.g., $H^{m}\left(X^{[k]}\right)$ 's).

\section{DeFormation THEORY}

Our basic reference is $[\mathrm{Pa}$, as summarized in [Fr2] for the normal crossing case and whose terminology and notations we shall generally follow 117 For $X$ either a compact analytic variety, or a germ of a reduced analytic variety, we shall denote by $\operatorname{Def}(X)$ the space parametrizing the corresponding family $X_{\operatorname{Def}(X)} \stackrel{\pi}{\rightarrow} \operatorname{Def}(X)$ that is versal for germs of flat families $X \stackrel{\pi}{\rightarrow} S$ with $\pi^{-1}\left(s_{0}\right)=X$. The Zariski tangent space to $\operatorname{Def}(X)$ is

$$
T_{X} \operatorname{Def}(X)=\mathbb{E x t}_{\mathcal{O}_{X}}^{1}\left(\Omega_{X}^{1}, \mathcal{O}_{X}\right) .
$$

\footnotetext{
${ }^{15}$ We will not try to explain this precisely, but note that in the Clemens-Schmid exact sequence the effects of doing a modification to $X$ cancel out and leave unchanged the terms with the limiting mixed Hodge structures. This phenomenon is of course familiar from Deligne's theory of mixed Hodge structures in which independence of the choice of smooth completions is established (cf. PS and the references cited therein).

${ }^{16}$ More precisely, to each is associated an equivalence class of limiting mixed Hodge structures. In [St1 it is shown that the two equivalence classes of limiting mixed Hodge structures agree.

${ }^{17}$ We note that the setting of log-analytic geometry is an alternate, and in many ways preferable, way to present this theory ( $\left.\left.\mathrm{cf}^{-} \mathrm{A}^{\dagger}\right],[\mathrm{KN}], \mathrm{KU}\right]$ and the references cited in these works). For example, in this context the central concept of $d$-semi-stability (cf. [I.6 below) simply becomes the existence of a log structure. Moreover, a logarithmic deformation of a smoothable normal crossing variety remains smoothable; none of the "bad" components in $\operatorname{Def}(X)$ can arise. In the setting of logarithmic deformation theory, unobstructed deformations of $X$ simply means inde-

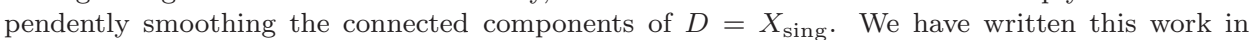
the traditional setting in part because this allows us more easily to connect with the other topics discussed.
} 
As usual we think of $\xi \in T_{X} \operatorname{Def}(X)$ as giving a family

$$
x_{\xi} \rightarrow \Delta_{\epsilon}
$$

where $\Delta_{\epsilon}=\operatorname{Spec} \mathbb{C}[\epsilon], \epsilon^{2}=0$.

Of basic importance for us will be the exact sequence

$$
\begin{aligned}
0 & \rightarrow H^{1}\left(\operatorname{Ext}_{\mathcal{O}_{X}}^{0}\left(\Omega_{X}^{1}, \mathcal{O}_{X}\right)\right) \rightarrow \operatorname{Ext}_{\mathcal{O}_{X}}^{1}\left(\Omega_{X}^{1}, \mathcal{O}_{X}\right) \\
& \rightarrow H^{0}\left(\operatorname{Ext}_{\mathcal{O}_{X}}^{1}\left(\Omega_{X}^{1}, \mathcal{O}_{X}\right)\right) \stackrel{\delta}{\rightarrow} H^{2}\left(\operatorname{Ext}_{\mathcal{O}_{X}}^{0}\left(\Omega_{X}^{1}, \mathcal{O}_{X}\right)\right)
\end{aligned}
$$

that results from the local to global spectral sequence for Ext. The image of the first map will be denoted by

$$
T_{X} \operatorname{Def}^{\mathrm{es}}(X) \rightarrow T_{X} \operatorname{Def}(X)
$$

it represents the Zariski tangent space to the equisingular, or locally trivial for the germs $X_{x}$ in $X$, deformations. For $x \in X$ the image of the map

$$
\operatorname{Ext}_{\mathcal{O}_{X}}^{1}\left(\Omega_{X}^{1}, \mathcal{O}_{X}\right) \rightarrow \operatorname{Ext}_{\mathcal{O}_{X}}^{1}\left(\Omega_{X}^{1}, \mathcal{O}_{X}\right)_{x}
$$

represents the $1^{\text {st }}$ order deformation of the germ $X_{x}$ of analytic variety induced by a global $1^{\text {st }}$ order deformation of $X$.

In our situation where $X$ is locally a product of normal crossing varieties given by (I.3) the local deformation theory is particularly harmonious. Taking first the case when $X$ is a germ of a normal crossing variety given locally in $\mathbb{C}^{n+1}$ by

$$
f(x)=: x_{1} \cdots x_{k}=0
$$

with versal deformation space $X \subset \mathbb{C}^{n} \times \mathbb{C}$ given by $f(x)=t$, and with the notations

$$
\begin{cases}X_{i}=\left\{x_{i}=0\right\}, & \\ D_{i}=X_{i} \cap\left(\bigcup_{j \neq i} X_{j}\right) & \text { defined by the ideal }\left\{\partial_{x_{i}} f=x_{1} \cdots \widehat{x_{i}} \cdots x_{k}\right\}, \\ D=\cup D_{i}=X_{\text {sing }} & \\ X^{[\ell]}=\coprod_{|I|=\ell} X_{I} & \text { where } I=\left(i_{1}, \ldots, i_{\ell}\right) \text { with } 1 \leqq i_{1}<\cdots<i_{\ell} \leqq n \\ & \text { and } X_{I}=X_{i_{1} \cap \cdots \cap X_{i_{\ell}}}\end{cases}
$$

we have as $\mathcal{O}_{X}$-modules

$$
\operatorname{Ext}_{\mathcal{O}_{X}}^{i}\left(\Omega_{X}^{1}, \mathcal{O}_{X}\right) \cong \begin{cases}\operatorname{ker}\left\{T_{X} \otimes \mathcal{O}_{X} \rightarrow \mathcal{O}_{X}(X)\right\} & i=0 \\ \left(I_{X} / I_{X}^{2}\right)^{*} \otimes \mathcal{O}_{D} & i=1 \\ 0 & i \geqq 2\end{cases}
$$

This follows from the Ext-sequence arising from the exact sequence

$$
0 \rightarrow I_{X} / I_{X}^{2} \rightarrow \Omega_{X}^{1} \otimes \mathcal{O}_{X} \rightarrow \Omega_{X}^{1} \rightarrow 0
$$

which, setting $\varphi_{i}=\partial_{x_{i}} f d x_{i}=x_{1} \cdots \widehat{x_{i}} \cdots x_{k} d x_{i}$, gives that $\Omega_{X}^{1}$ is freely generated over $\mathcal{O}_{X}$, and that $\Omega_{X}^{1}$ is generated by $d x_{1}, \ldots, d x_{n+1}$ subject to the defining relation

$$
d f=\sum_{i} \varphi_{i}=0 .
$$

Assuming now that $X$ is a complete algebraic variety that is locally a normal crossing variety given locally by (II.2), motivated by the middle equation in (II.3) and taking into account the scaling of $f$ under $f \rightarrow u f$ where $u \in \mathcal{O}_{X}^{*}$ and following [Fr2], we may define the infinitesimal normal bundle by

$$
\mathcal{O}_{D}(X)=\operatorname{Ext}_{\mathcal{O}_{X}}^{1}\left(\Omega_{X}^{1}, \mathcal{O}_{X}\right) .
$$


The point here is that, unless we are given a global embedding of $X$ as a hypersurface in a smooth variety $X$, we cannot define the normal bundle $\mathcal{O}_{X}(X)$, but we are able to intrinsically define what would be the restriction to $D$ of the normal bundle of $X$ in a smooth ambient space if such exists.

In more detail, we set

$$
\begin{aligned}
\mathcal{O}_{D_{i}}(-X) & =\left(I_{D_{i}} / I_{D_{i}}^{2}\right) \otimes_{\mathcal{O}_{D_{i}}}\left(I_{X_{i}} / I_{X_{i}} I_{D_{i}}\right) \\
\mathcal{O}_{D}(-X) & =\left(I_{X_{1}} / I_{X_{1}} J_{D}\right) \otimes_{\mathcal{O}_{D}} \cdots \otimes_{\mathcal{O}_{D}}\left(I_{X_{k}} / I_{X_{k}} J_{D}\right)
\end{aligned}
$$

where $I_{X_{i}}$ is the ideal sheaf of $X_{i}$ in $X$ and $J_{D_{i}}$ is the ideal sheaf of $D_{i}$ in $X$. The second equation then serves to define $\mathcal{O}_{D}(X)$ in agreement with (II.5) and we have

$$
\operatorname{Ext}_{\mathcal{O}_{X}}^{1}\left(\Omega_{X}^{1}, \mathcal{O}_{X}\right) \cong \mathcal{O}_{D}(-X)^{*} \otimes \mathcal{O}_{D}
$$

where $\mathcal{O}_{D}(-X)=I_{X} / I_{X}^{2}$ in case we have $X \subset X$.

As in [Fr2], $X$ is said to be $d$-semi-stable if

$$
\mathcal{O}_{D}(X) \cong \mathcal{O}_{D},
$$

that is there exists a nowhere vanishing section of the line bundle $\mathcal{O}_{D}(X)$ over $D 18$

We shall assume throughout that $X$ is d-semi-stable.

Returning to (II.1) and using (II.5) we have the map

$$
\mathbb{E x t}_{\mathcal{O}_{X}}^{1}\left(\Omega_{X}^{1}, \mathcal{O}_{X}\right) \rightarrow H^{0}\left(\mathcal{O}_{D}(X)\right)
$$

which we denote by $\xi \rightarrow \xi_{D}$ where $\xi \in \mathbb{E x t}_{\mathcal{O}_{X}}^{1}\left(\Omega_{X}^{1}, \mathcal{O}_{X}\right)=T_{X} \operatorname{Def}(X)$. We shall say that the $1^{\text {st }}$ order deformation $\xi$ of $X$ is smoothing if $\xi_{D}$ is nowhere vanishing. We shall also generally abuse terminology by dropping the "1 $1^{\text {st }}$ order", and we shall say that $\xi_{D}$ is non-zero rather than nowhere vanishing. Given such a $\xi$ we have a family $X_{\xi} \rightarrow \Delta_{\epsilon}$, where $\Delta_{\epsilon}=\operatorname{Spec} \mathbb{C}[\epsilon]$ with $\epsilon^{2}=0$, where $X_{\xi}$ is smooth and in which the fibre over 0 is $X$. As noted earlier, it may or may not be the case that $x_{\xi} \rightarrow \Delta_{\epsilon}$ can be lifted to a family $X \rightarrow \Delta$; this issue will play no role in what follows.

For later reference we note that the sheaf $\Omega_{X}^{1}$ of Kähler differentials is defined by (II.4), where the injectivity of the first map is a property of $X$ as given by (II.2). It is not locally free as a sheaf of $\mathcal{O}_{X}$-modules, but rather has a torsion subsheaf

$$
\tau_{X}^{1} \subset \Omega_{X}^{1}
$$

which is locally generated by the forms $\varphi_{i}$ define above. Its support is $D=X_{\text {sing }}$, and as noted in [Fr2] since $\Omega_{X}^{1} / \tau_{X}^{1}$ is locally free the above inclusion induces an isomorphism

$$
\operatorname{Ext}_{\mathcal{O}_{X}}^{1}\left(\Omega_{X}^{1}, \mathcal{O}_{X}\right) \stackrel{\sim}{\rightarrow} \operatorname{Ext}_{\mathcal{O}_{X}}^{1}\left(\tau_{X}^{1}, \mathcal{O}_{X}\right),
$$

which "explains" the identification (II.5).

Of importance for this work will be to consider the set $A$ of connected components $D_{a}, a \in A$, of $D$. Recalling our blanket assumption that there exists a $1^{\text {st }}$ order smoothing deformation of $X$, we will have for each $\alpha \in A$

$$
\mathcal{O}_{D_{\alpha}}(X) \cong \mathcal{O}_{D_{\alpha}}
$$

where the particular isomorphism depends on the choice of a non-zero section $\xi$ of $\mathcal{O}_{D}(X)$. Thus if $\xi \in T_{X} \operatorname{Def}(X)$ with restriction $\xi_{D_{a}}$ to $\mathcal{O}_{D_{\alpha}}(X)$, we see that

\footnotetext{
${ }^{18}$ Here the point is that if we have $X \subset X$ with $X$ smooth and $X \stackrel{\pi}{\rightarrow} \Delta$ with $\pi^{-1}(0)=X$, then the conormal bundle $\mathcal{O}(-X)=I_{X} / I_{X}^{2}$ is trivial. Thus if we just have $X \subset \mathcal{X}$ where $\mathcal{X}$ is smooth, a necessary condition for there to exist $X \stackrel{\pi}{\rightarrow} \Delta$ as above is that $\mathcal{O}_{X}(X) \cong \mathcal{O}_{X}$. The $d$-semi-stability condition (I.6) is intrinsic to $X$ and does not require the existence of an $X$.
} 
along the component $D_{\alpha}$ of $D=X_{\text {sing }}$ the deformation of $X$ given by $\xi$ is either everywhere smoothing or equisingular. For each subset $B \subset A$ we set

$$
T_{X}^{B} \operatorname{Def}(X)=\left\{\xi \in T_{X} \operatorname{Def}(X): \xi_{D_{\beta}}=0 \text { for } \beta \in B\right\} .
$$

Then $T_{X}^{B} \operatorname{Def}(X)$ corresponds to the deformations that are equisingular along the $D_{\beta}$ for $\beta \in B$. The extremes are

- $B=\emptyset$ corresponds to the open set $T_{X}^{0} \operatorname{Def}(X)$ of smoothing deformations;

- $B=A$ corresponds to the space $T_{X} \operatorname{Def}^{\text {es }}(X)$ of equisingular deformations.

In a way that will be explained in detail later, this gives a stratification of $T_{X} \operatorname{Def}(X)$ and leads to the definition of the cone $\sigma_{X}$ mentioned in the introduction.

Example: A simple example is when $X$ is a nodal curve. The surjectivity of the map

$$
\operatorname{Ext}_{\mathcal{O}_{X}}^{1}\left(\Omega_{X}^{1}, \mathcal{O}_{X}\right) \rightarrow \underset{\alpha \in A}{\oplus} H^{0}\left(\mathcal{O}_{D_{\alpha}}(X)\right) \rightarrow 0
$$

corresponds to individually smoothing the nodes.

Example: Suppose that $X_{0}$ is a singular variety with isolated singular points $p_{\alpha}$ given by $f_{\alpha}(x)=0$. We may resolve the singularities to obtain $X$ where $D$ has connected components $D_{\alpha}$. The versal deformation spaces given by $f_{\alpha}(x)=t_{\alpha}$ for the germ of $X_{\alpha}$ at $p_{\alpha}$ and for the inverse image $X_{\alpha}$ of $p_{\alpha}$ in $X$ coincide (cf. $\mathrm{Pa}$ ). The failure of surjectivity of the first map in

$$
\mathbb{E x t}_{\mathcal{O}_{X}}^{1}\left(\Omega_{X}^{1}, \mathcal{O}_{X}\right) \rightarrow \underset{\alpha \in A}{\oplus} H^{0}\left(\mathcal{O}_{D_{\alpha}}(X)\right) \stackrel{\delta}{\rightarrow} H^{2}\left(\operatorname{Ext}_{\mathcal{O}_{X}}^{0}\left(\Omega_{X}^{1}, \mathcal{O}_{X}\right)\right)
$$

measures the obstruction to simultaneously smoothing the $p_{\alpha} \in X_{0}$.

When the $p_{\alpha}$ are ordinary double points the dual to the mapping $\delta$ in the sequence may be computed and leads to the conditions on the simultaneous smoothing of the nodes that may be lifted to a smoothing of $X 19$

In general when $X$ may be smoothed but the connected components $D_{\alpha}$ may not be independently smoothed, the situation is more complicated and necessitates the blowing up of $X$. This is the situation where in the setting of logarithmic deformation theory there are obstructions and will be discussed at another time.

The example of K3 surfaces is discussed in $\mathrm{Fr} 1$, Fr2 and $\mathrm{KN}$.

We next turn to the local case where the germ of variety $X$ is a product

$$
X=X^{1} \times \cdots \times X^{k}=\prod_{\mu \in U} X^{\mu}
$$

of normal crossing varieties 20 Letting $\pi_{\mu}: X \rightarrow X^{\mu}$ denote the projection, from the isomorphism of $\mathcal{O}_{X}$-modules

$$
\Omega_{X}^{1} \cong \oplus \pi_{\mu}^{*} \Omega_{X^{\mu}}^{1}
$$

leading to

$$
\operatorname{Ext}_{\mathcal{O}_{X}}^{1}\left(\Omega_{X}^{1}, \mathcal{O}_{X}\right) \cong \underset{\mu}{\operatorname{Ext}_{\mathcal{O}_{X}{ }^{\mu}}^{1}}\left(\Omega_{X^{\mu}}^{1}, \mathcal{O}_{X^{\mu}}\right) \otimes \mathcal{O}_{X}
$$

\footnotetext{
${ }^{19}$ If the mapping (II.7) is surjective, then

$$
\sigma_{X} \otimes \mathbb{R} \cong \operatorname{span}_{\mathbb{R}>0}\left\{i \xi_{D_{1}}, \ldots, i \xi_{(A)}\right\} .
$$

The reason for the " $i$ " in $i \xi_{D_{a}}$ is that if we think of $\xi_{D_{a}}$ as giving a tangent vector to a one parameter family then $i \xi_{a}$ is supposed to suggest turning around the origin - i.e., monodromy - in the family.

${ }^{20}$ This case is treated in [Fu1] and $\mathrm{Fu} 2$.
} 
we may extend the local theory in the evident way. The sequence (II.4) now becomes

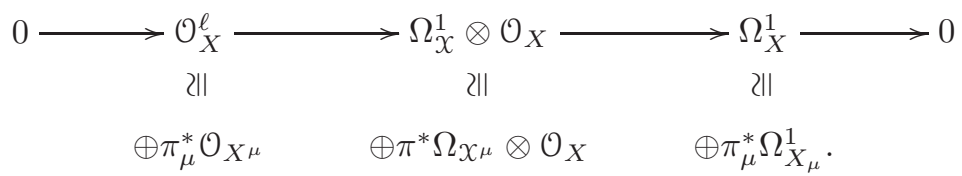

For

$$
\left\{\begin{array}{l}
D^{\mu}=X^{1} \times \cdots \times D^{\mu} \times \cdots \times X^{k} \\
D=\sum D^{\mu}=X_{\text {sing }}
\end{array}\right.
$$

we have

$$
\operatorname{Ext}_{\mathcal{O}_{X}}^{1}\left(\Omega_{X}^{1}, \mathcal{O}_{X}\right) \cong \oplus_{\mu} \pi_{\mu}^{*} \mathcal{O}_{D^{\mu}} .
$$

The local versal deformation space is the product of the local versal deformation spaces for the factors.

As in the normal crossing case one may intrinsically define an infinitesimal normal sheaf $\mathcal{N}$. In the stratification $X_{\text {sing, } \ell}$ of $X_{\text {sing }}$ by the number of singular factors in the local product of normal crossing varieties description given by (I.1), $\mathcal{N}$ is a coherent sheaf whose restriction to $X_{\mathrm{sing}, \ell} \backslash X_{\mathrm{sing}, \ell+1}$ is locally free of rank $\ell$. The definition of $d$-semi-stability may then be extended. This will be done in the work in progress; the practical effect of assuming $d$-semi-stability is that the to be constructed locally defined sheaves of $\mathcal{O}_{X}$-modules $\Omega_{X_{\xi}}^{1} \otimes \mathcal{O}_{X}$ and $\Omega_{X_{\xi} / \Delta \epsilon}^{1}(\log X) \otimes \mathcal{O}_{X}$ patch together to give global sheaves over all of $X$.

When we consider the global situation where $X$ is locally a product as above, we retain our standing assumption that in the map

$$
\mathbb{E x t}_{\mathcal{O}_{X}}^{1}\left(\Omega_{X}^{1}, \mathcal{O}_{X}\right) \rightarrow H^{0}\left(\operatorname{Ext}_{\mathcal{O}_{X}}^{1}\left(\Omega_{X}^{1}, \mathcal{O}_{X}\right)\right)
$$

there is $\xi \in \mathbb{E x t}_{\mathcal{O}_{X}}^{1}\left(\Omega_{X}^{1}, \mathcal{O}_{X}\right)$ which is a smoothing deformation along each component of $X_{\text {sing. }}$. This does not mean that for each germ $X_{x} \subset X$ the global deformations map onto the space of local smoothings of $X_{x}$. It does mean that there is a $\xi \in \mathbb{E x t}_{\mathcal{O}_{X}}^{1}\left(\Omega_{X}^{1}, \mathcal{O}_{X}\right)$ whose local image in each

$$
\operatorname{Ext}_{\mathcal{O}_{X^{\mu}}}^{1}\left(\Omega_{X^{\mu}}^{1}, \mathcal{O}_{X^{\mu}}\right) \cong \mathcal{O}_{D^{\mu}}\left(X^{\mu}\right)
$$

is non-vanishing. Then the above discussion regarding the connected components of $D$ extends and will be taken up in a future work.

One significant difference in the local situation where the number $l$ of local factors is strictly larger than one is this: For a 1-parameter smoothing family

$$
x_{\Delta} \rightarrow \Delta
$$

with tangent $\xi$, the total space $X_{\Delta}$ is singular. This can be seen already in the local situation

$$
\left\{\begin{array}{l}
x y=t_{1} \\
u v=t_{2}
\end{array}\right.
$$

where the disc is given by $t_{1} / t_{2}=\lambda \neq 0$. Then even though the total space $X \rightarrow \Delta_{1} \times \Delta_{2}$ is smooth, the subvariety $X_{\Delta} \subset X$ is singular at the origin.

A final comment for this section: In the study of varieties that are locally products of normal crossing varieties, the necessary multi-index notations may obscure 
the essential points. Our experience has been that for normal crossings the two cases

$$
\left\{\begin{array}{l}
x y=0 \\
u v w=0
\end{array}\right.
$$

and for products of normal crossings the cases

$$
x y=0, \quad u v=0
$$

capture all the essential phenomena. The main subtlety seems to arise when we smooth the singularity to obtain $X$, various exact sequences over $\mathcal{O}_{X}$ fail to become exact when we restrict to $X$ by tensoring with $\mathcal{O}_{X}$ and some care must be taken in the computations to keep track of this.

\section{Proofs of Theorems I And I'}

This initial discussion is mainly local. We begin with a germ of normal crossing variety $X$ given by (11.2). Given a non-zero

$$
\xi \in \operatorname{Ext}_{\mathcal{O}_{X}}^{1}\left(\Omega_{X}^{1}, \mathcal{O}_{X}\right) \cong \mathcal{O}_{D}(X)
$$

we denote by $x_{\xi} \stackrel{\pi}{\rightarrow} \Delta_{\epsilon}$ the corresponding versal family

$$
x_{1} \cdots x_{k}=\epsilon, \quad \epsilon^{2}=0
$$

and write the extension as

$$
0 \rightarrow \Omega_{\Delta_{\epsilon}}^{1} \otimes \mathcal{O}_{X} \rightarrow \Omega_{X_{\xi}}^{1} \otimes \mathcal{O}_{X} \rightarrow \Omega_{X}^{1} \rightarrow 0
$$

Here, $\mathcal{O}_{X_{\xi}}$ is locally isomorphic to $\mathcal{O}_{X}[\epsilon]$ and $\Omega_{X_{\xi}}^{1}$ is the free $\mathcal{O}_{X_{\xi}}$-module generated by $d x_{1}, \ldots, d x_{n+1}, d \epsilon$ modulo the relation $d \epsilon=\sum_{i=1}^{k} \varphi_{i}$. Unless otherwise noted the tensor products are over $\mathcal{O}_{x_{\xi}}$. We are setting $\Omega_{\Delta_{\epsilon}}^{1}=\pi^{*} \Omega_{\Delta_{\epsilon}}^{1}$ and are writing the sequence in this way to emphasize the scaling property with respect to $\xi$. Note that $\Omega_{X_{\xi}}^{1} \otimes \mathcal{O}_{X}$ is the $\mathcal{O}_{X}$-module with the same set of generators and defining relation, and where in computations we set $x_{1} \cdots x_{k}=0$ but do not set $d\left(x_{1} \cdots x_{k}\right)=$ $\sum_{i=1}^{k} \varphi_{i}=0$.

We may as usual define the free $\mathcal{O}_{X_{\xi}}$-module $\Omega_{X_{\xi}}^{1}(\log X)$ with generators $d x_{1} / x_{1}$, $\ldots, d x_{k} / x_{k}, d x_{k+1}, \ldots, d x_{n+1}, d \epsilon / \epsilon$ modulo the relation $d \epsilon / \epsilon=\sum_{i=1}^{k} d x_{i} / x_{i}$. Then

$$
\Omega_{X_{\xi}}^{1}(\log X) \otimes \mathcal{O}_{X}
$$

is freely generated over $\mathcal{O}_{X}$ with the same set of generators and defining relation.

We next define

$$
\Omega_{X_{\xi} / \Delta_{\epsilon}}^{1}(\log X) \otimes \mathcal{O}_{X}
$$

to be the $\mathcal{O}_{X}$-module with the above generators and generating relation

$$
\sum_{i} d x_{i} / x_{i}=0
$$

We will describe this intrinsically in a moment. Here we note the crucial point that in the case of a global normal crossing variety $X$ fixing a nowhere zero $\xi \in$ $\operatorname{Ext}_{\mathcal{O}_{X}}^{1}\left(\Omega_{X}^{1}, \mathcal{O}_{X}\right)$ uniquely locally determines a normalized generator $\epsilon-x_{1} \cdots x_{k}$ of the ideal $I_{x_{\xi}}$ of $X_{\xi}$ : If we have $x_{i}^{\prime}=u_{i} x_{i}$ where $u_{i} \in \mathcal{O}_{X}^{*}$, it follows from the equality

$$
\epsilon-x_{1} \cdots x_{k}=\epsilon-x_{i}^{\prime} \cdots x_{k}^{\prime}
$$


of normalized generators that $u=u_{1} \cdots u_{k}=1$ so that $\sum d x_{i}^{\prime} / x_{i}^{\prime}=\sum d x_{i} / x_{i}$. This gives

(III.1) may be defined by the pair $(X, \xi)$ where $X$ is a local normal crossing variety and $\xi \in T_{X} \operatorname{Def}(X)$ is non-vanishing 21

One small point to notice is that the natural map

$$
\pi^{*} \Omega_{\Delta}^{1} \otimes \mathcal{O}_{X} \rightarrow \pi^{*} \Omega_{\Delta}^{1}(\log 0) \otimes \mathcal{O}_{X}
$$

is zero; this is because

$$
d \epsilon \otimes 1 \rightarrow \epsilon\left(\frac{d \epsilon}{\epsilon}\right) \otimes 1=\frac{d \epsilon}{\epsilon} \otimes \epsilon=0 .
$$

A related point is that there is a natural map of $\mathcal{O}_{X}$-modules

$$
\Omega_{X}^{1} \rightarrow \Omega_{x_{\xi} / \Delta_{\epsilon}}^{1}(\log X) \otimes \mathcal{O}_{X}
$$

given on generators by $d x_{i} \rightarrow d x_{i}$, and then this map has kernel $\tau_{X}^{1}$. Since $\Omega_{X_{\xi} / \Delta_{\epsilon}}^{1}(\log X) \otimes \mathcal{O}_{X}$ is locally free we know that the above map must have kernel containing $\tau_{X}^{1}$; computation shows that equality holds.

We follow the usual notations

$$
\begin{array}{ll}
X^{[1]}=\coprod_{i} X_{i}=\tilde{X} & \text { (normalization of } X \text { ) } \\
X^{[2]}=\coprod_{i<j} X_{i} \cap X_{j}=\widetilde{X}_{\text {sing }} & \text { (normalization of } X_{\text {sing }} \text { ) } \\
X^{[3]}=\coprod_{i<j<k} X_{i} \cap X_{j} \cap X_{k} &
\end{array}
$$

with maps

and where we set $a_{1}=a: \widetilde{X} \rightarrow X$.

$$
a_{j}: X^{[j]} \rightarrow X
$$

(III.3) Proposition (Basic Diagram): We have

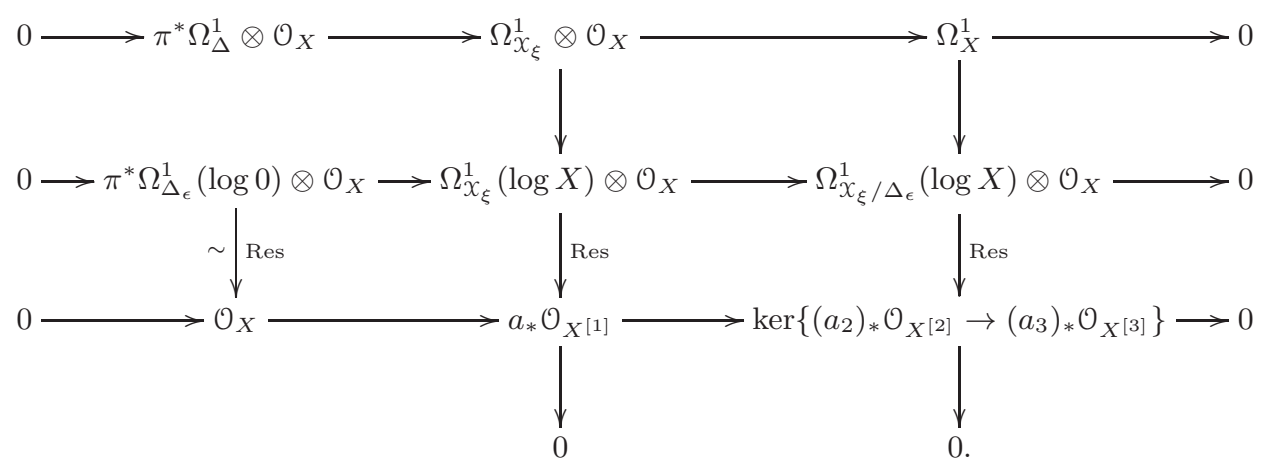

The right-hand map in the bottom row is the truncation of a resolution of $\mathcal{O}_{X}$ that is given in the comment immediately following the proof of this proposition.

\footnotetext{
${ }^{21}$ In the setting of logarithmic geometry, to define a logarithmic structure on a normal crossing variety requires $d$-stability, and then the variety is log-smooth ( $\underline{\mathrm{St} 2}, \mathrm{KN}]$ ).
} 
Proof. We begin with the standard diagram of $\mathcal{O}_{x_{\xi}}$-modules

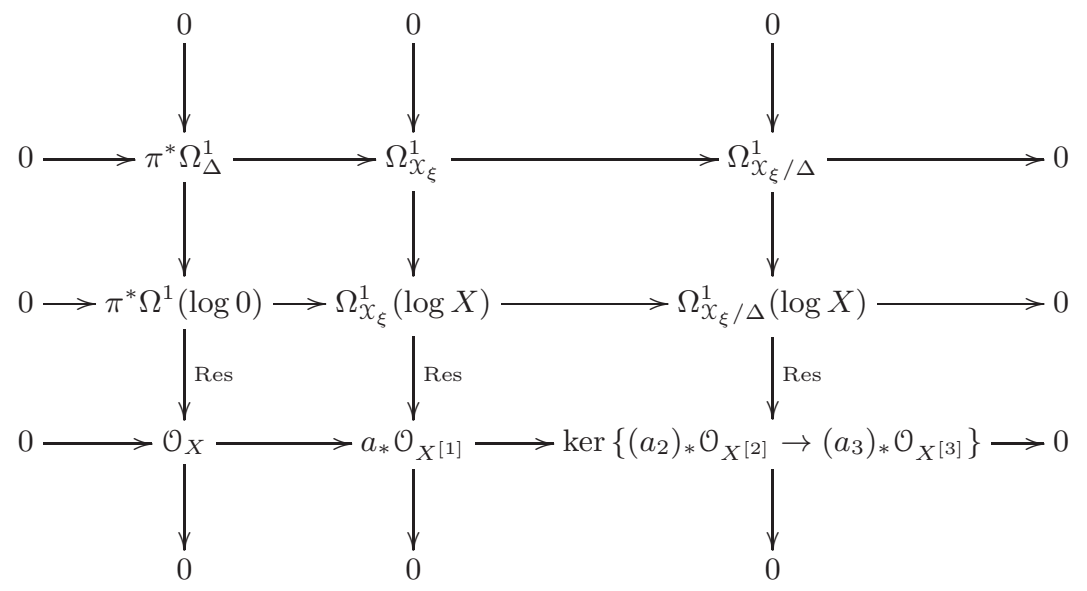

where the $\mathcal{O}_{X}$-modules on the bottom row are considered as $\mathcal{O}_{X_{\xi}}$-modules by the restriction map $\mathcal{O}_{X_{\xi}} \rightarrow \mathcal{O}_{X}$. When we restrict to $X$ by

$$
\left.\mathcal{F} \rightarrow \mathcal{F}\right|_{X}=\mathcal{F} \otimes_{\mathcal{O}_{X}} \mathcal{O}_{X}
$$

for an $\mathcal{O} x$-module $\mathcal{F}$, then as noted above we may lose exactness in certain places. Calculations in local coordinates gives the exactness in the basic diagram, where we note that

$$
\Omega_{X_{\xi} / \Delta_{\epsilon}}^{1} \otimes \mathcal{O}_{X}=\Omega_{X}^{1}
$$

as the top row reduces to the defining relation $0 \rightarrow I_{X} / I_{X}^{2} \stackrel{d}{\rightarrow} \Omega_{X_{\xi}}^{1} \otimes \mathcal{O}_{X} \rightarrow \Omega_{X}^{1} \rightarrow 0$ for Kähler differentials.

To give the flavor of the calculations we consider the simplest non-trivial case of $x y=\epsilon$. Then

- $\Omega_{X_{\xi}}^{1} \otimes \mathcal{O}_{X}$ is generated as an $\mathcal{O}_{X}$-module by $d x, d y, d \epsilon$ with the defining relation $x d y+y d x=d \epsilon$;

- $\Omega_{X_{\xi}}^{1}(\log X) \otimes \mathcal{O}_{X}$ is generated by $d x / x, d y / y, d \epsilon / \epsilon$ with the defining relation $d x / x+d y / y=d \epsilon / \epsilon$.

Any $\omega \in \Omega_{X_{\varepsilon}}^{1} \otimes \mathcal{O}_{X}$ is of the form $f(x, y) d x+g(x, y) d y$, and using that $\otimes \mathcal{O}_{X}$ means setting " $x y=0$ " we see that $\omega$ may be normalized to be

$$
\omega=\left(f_{1}(x)+f_{2}(y)\right) d x+\left(g_{1}(y)+g_{2}(y)\right) d y, \quad f_{1}(0)=g_{2}(0)=0 .
$$

Similarly, $\varphi \in \Omega_{X_{\xi}}^{1}(\log X) \otimes \mathcal{O}_{X}$ may be normalized to be

$$
\varphi=\left(a_{1}(x)+a_{2}(y)\right) \frac{d x}{x}+\left(b_{1}(x)+b_{2}(y)\right) \frac{d y}{y}, \quad a_{1}(0)=b_{2}(0)=0 .
$$

Then

$$
\operatorname{Res} \varphi=a_{2}(y) \oplus b_{1}(x) \in\left(a_{*}\right) \mathcal{O}_{X^{[1]}} .
$$

If $\operatorname{Res} \varphi=0$, then writing $a_{1}(x)=x \widetilde{a_{1}}(x)$ and $b_{2}(y)=y \widetilde{b_{2}}(y)$ we have

$$
\varphi=\widetilde{a_{1}}(x) d x+\widetilde{b_{2}}(y) d y \in \Omega_{X_{\xi}}^{1} \otimes \mathcal{O}_{X} .
$$

A similar calculation gives the exactness of the right-hand column. For the case of a triple point $x y z=\epsilon$ the residue calculation is more complicated and is similar to $(2.10)$ in $\mathrm{Fr} 2$. 
We want to make two comments on the basic diagram. The first is

The bottom row in the basic diagram is the truncation of the resolution

$$
\text { of } \mathcal{O}_{X} . \quad 0 \rightarrow \mathcal{O}_{X} \rightarrow\left(a_{1}\right)_{*} \mathcal{O}_{X}[1] \rightarrow\left(a_{2}\right)_{*} \mathcal{O}_{X^{[2]}} \rightarrow\left(a_{3}\right)_{*} \mathcal{O}_{X^{[3]}} \rightarrow \cdots
$$

The map $\left(a_{k}\right)_{*} \mathcal{O}_{X^{[k]}} \rightarrow\left(a_{k+1}\right)_{*} \mathcal{O}_{X^{[k+1]}}$ is given by

$$
u_{i_{1} \cdots i_{k}} \text { on } X_{i_{1}} \cap \cdots \cap X_{i_{k}}
$$

maps to

$$
\left.\sum_{j}(-1)^{j+1} u_{i_{1} \cdots \hat{i}_{j} \cdots i_{k+1}}\right|_{X_{i_{1}} \cap \cdots \cap X_{i_{k+1}}} .
$$

This is standard (cf. [Fr2], [St1] and [Zu]). We note also the resolution

$$
0 \rightarrow \mathbb{C}_{X} \rightarrow\left(a_{1}\right)_{*} \mathbb{C}_{X^{[1]}} \rightarrow\left(a_{2}\right)_{*} \mathbb{C}_{X^{[2]}} \rightarrow\left(a_{3}\right)_{*} \mathbb{C}_{X^{[3]}} \rightarrow \cdots
$$

of the constant sheaf on $X$.

The second is that we list the main take-aways from the basic diagram:

(i) Given $X$ and $\xi \in \mathbb{E x t}_{\mathcal{O}_{X}}^{1}\left(\Omega_{X}^{1}, \mathcal{O}_{X}\right)$ with the property that $\xi$ is non-zero along $D$, we may by definition construct an extension of $\mathcal{O}_{X}$-modules

$$
0 \rightarrow \mathcal{O}_{X} \rightarrow \mathcal{F}_{\xi} \rightarrow \Omega_{X}^{1} \rightarrow 0 .
$$

(ii) From $\left[\mathrm{Pa}\right.$, we may actually construct a space $X_{\xi}$ with structure sheaf $\mathcal{O}_{X_{\xi}}$ locally isomorphic to $\mathcal{O}_{X}[\epsilon]$ giving a mapping $X_{\xi} \rightarrow \Delta_{\epsilon}=$ Spec $\mathbb{C}[\epsilon]$ where

- $X_{\xi}$ is smooth (this is the assumption that $\xi_{D} \neq 0$ );

- $\mathcal{F}_{\xi} \cong \Omega_{X_{\xi}}^{1} \otimes \mathcal{O}_{X}$ as $\mathcal{O}_{X}$-modules; this is the top row in the basic diagram.

(iii) We may then proceed, using $\Omega_{x_{\xi}}^{1}$ as an $\mathcal{O}_{x_{\xi}}$-module, to construct the remainder of the basic diagram; the inclusion map $\mathcal{O}_{X} \rightarrow \Omega_{X_{\xi}}^{1}(\log X) \otimes \mathcal{O}_{X}$ is given by $1 \rightarrow d \epsilon / \epsilon$, and then the quotient defines the $\mathcal{O}_{X}$-module $\Omega_{X_{\xi} / \Delta_{\epsilon}}^{1}(\log X) \otimes \mathcal{O}_{X}$.

(iv) From this we may, in the standard way, proceed to construct the complex $\left(\Omega_{X_{\xi} / \Delta_{\epsilon}}(\log X) \otimes \mathcal{O}_{X}, d\right)$; as will be noted below, the hypercohomology of this complex will give the complex vector space $V_{\xi}$ and Hodge filtration $F_{\xi}^{\bullet}$ for the limiting mixed Hodge structure.

(v) The previous steps are either explicit or implicit in [Fr2]; the final steps to define the weight filtration and $\mathbb{Q}$-structure may then be carried out by the methods in [St2.

We will elaborate more on this at the end of the section.

Turning now to the case where $X$ is locally a product of normal crossing varieties as given by (I.2), we may extend the discussion above with one significant change. Namely, in the local situation instead of a single smoothing deformation $\xi \in T_{X}^{0} \operatorname{Def}(X)$ we now need to be given a $k$-tuple

$$
\boldsymbol{\xi}=\left(\xi_{1}, \ldots, \xi_{k}\right)
$$

where $\xi_{i}$ smooths the factor $X_{i}$ in $X$. Then for $\lambda=\left(\lambda_{1}, \ldots, \lambda_{k}\right)$ with all $\lambda_{i} \neq 0$

$$
\boldsymbol{\xi}_{\lambda}=: \sum \lambda_{i} \xi_{i} \in T_{X} \operatorname{Def}(X)
$$

is a smoothing deformation of $X$. This is all local.

Globally we need to be given an $\ell$-tuple $\xi \in \stackrel{\ell}{\oplus} T_{X} \operatorname{Def}(X)$ such that locally around each $x \in X$ there is a $k$ sub-tuple of $\boldsymbol{\xi}$ that satisfies the above condition. 
We note again the difference when the number of local factors $k \geqq 2$; if $\boldsymbol{\xi}_{\lambda}$ is tangent to a family $X_{\Delta_{\lambda}} \rightarrow \Delta_{\lambda}$, then

$$
\text { the total space } x_{\Delta_{\lambda}} \text { is singular. }
$$

These singularities are of a standard form and may be resolved to give a standard family

where $\tilde{x}_{\Delta_{\lambda}}$ is smooth.

$$
\widetilde{x}_{\Delta_{\lambda}} \rightarrow \widetilde{\Delta}_{\lambda}
$$

Setting $\Delta_{\epsilon_{j}}=\operatorname{Spec} \mathbb{C}\left[\epsilon_{j}\right]$ and $\Delta_{\epsilon}=\prod_{j} \Delta_{\epsilon_{j}}$, using the projection $X_{1} \times \cdots \times X_{k} \stackrel{\pi_{j}}{\longrightarrow}$ $X_{j}$, we define

$$
\Omega_{X_{\xi} / \Delta_{\epsilon}}^{1}(\log X) \otimes \mathcal{O}_{X}=\oplus \pi_{j}^{*} \Omega_{X_{j} / \Delta_{\epsilon_{j}}}^{1}\left(\log X_{j}\right) \otimes \mathcal{O}_{X} .
$$

In coordinates, for the case where $X$ is given by

$$
\left\{\begin{array}{l}
x y=t_{1} \\
u v=t_{2}
\end{array}\right.
$$

so that $I_{X}$ is generated by $x y$ and $u v, \Omega_{X_{\xi} / \Delta_{\epsilon}}^{1}(\log X) \otimes \mathcal{O}_{X}$ is generated as an $\mathcal{O}_{X}$-module by $d x / x, d y / y, d u / u, d v / v$ with the relations $d x / x+d y / y=0, d u / u+$ $d v / v=0$. This coordinate description extends in the evident way when $X$ is given by (1.2).

Finally, we will relate this construction to that given in [Fu1], [Fu2. We have

$$
x \stackrel{\pi}{\rightarrow} S
$$

where locally in $\mathbb{C}^{n+k}$ with coordinates $\left(x_{1}, \ldots x_{n}, t_{1}, \cdots, t_{k}\right)$ and using the notation (1.2), $X$ is given by

$$
\left\{\begin{array}{c}
x_{I_{1}}=t_{1} \\
\vdots \\
x_{I_{k}}=t_{k}
\end{array}\right.
$$

and $\pi$ is the projection $(\mathbf{x}, \mathbf{t}) \rightarrow \mathbf{t}$. There are then normal crossing divisors $y \subset X$ and $T \subset S$ such that (III.7) is a map

$$
(X, y) \rightarrow(S, T)
$$

as defined in Fu1, Fu2. If $\operatorname{dim} X=n$ and $\operatorname{dim} S=\ell$, then $\operatorname{dim} X=n+\ell$. Locally $S$ is embedded in $\mathbb{C}^{k} \times \mathbb{C}^{\ell-k}$ where the first $k$ coordinates are the $t_{i}$ above and the remaining $\ell-k$ coordinates are parameters. We note that $\pi^{-1}$ (set of coordinate hyperplanes in $\mathbb{C}^{k}$ ) is a singular subvariety of $X$. Globally, we will have divisors $D_{1}, \ldots, D_{\ell}$ on $X$ such that locally $D_{1}, \ldots D_{k}$ are the inverse image under $\pi$ of the coordinate hyperplanes $t_{i}=0$ and $y=D_{1}+\cdots+D_{\ell}$ is a reduced normal crossing divisor in $\mathcal{X}$ with $X=D_{1} \cap \cdots \cap D_{\ell}$.

Discussion of the proofs of Theorems 1 and $\sqrt{1^{2} 2}$ For the case when $X$ is a local normal crossing variety, using (III.4) the essentials of the proof are in [Fr2 and St2. The sheaves

$$
\wedge_{\dot{\xi}}^{\bullet}=\wedge^{\bullet} \Omega_{X_{\xi} / \Delta_{\epsilon}}^{1}(\log X) \otimes \mathcal{O}_{X}
$$

\footnotetext{
${ }^{22}$ In a work in progress we intend to provide details for this argument with emphasis on the local structure and how this relates to the results in [CK and CKS1.
} 
form a filtered complex in the evident way, and

$$
\left\{\begin{array}{l}
V_{\xi}=\mathbb{H}^{m}\left(\wedge_{\xi}^{\bullet}\right) \\
F^{\bullet} V_{\xi}=F^{\bullet} \mathbb{H}^{m}\left(\wedge_{\xi}^{\bullet}\right)
\end{array}\right.
$$

defines the vector space and Hodge filtration for the limiting mixed Hodge structure. As usual, dating to [St1] (cf. also [Zu]), the construction of the monodromy weight filtration and $\mathbb{Q}$-structure are more subtle. These may be carried out by an adaptation of the methods in $\S 5$ in $\mathrm{St2}$.

More specifically, in [St1] and $[\mathrm{Zu}]$ associated to a standard family $X \rightarrow \Delta$ several cohomological mixed Hodge complexes are constructed. One of these, denoted there by $A^{\bullet}$ (recalled in the proof of Theorem (VI) in Section IV below) leads to the limit mixed Hodge structure. Another of these, denoted by $L^{\bullet}$ in loc. cit., leads to the mixed Hodge structures on $H^{*}(X \backslash X)$ and on $H^{*}(X, X \backslash X)$. In [St2], in the setting of $\log$ geometry which in his Section 5 corresponds to our $(X, \xi)$, the analogue of $L^{\bullet}$, denoted there by $K^{\bullet}$, is constructed. Analysis of the construction leads to a cohomological mixed complex in our $(X, \xi)$ setting that gives a limiting mixed Hodge structure on $V_{\xi}$.

For the general case where the central fibre $X$ in a global map (III.7) is given locally by (III.8), in Fu1, Fu2 the methods of [St1 are extended to show that for $s \in S$ and $X_{s}=\pi^{-1}(s)$ the hypercohomology of the complexes $\Omega_{X / S}^{\bullet}(\log y) \otimes \mathcal{O}_{X_{s}}$ give mixed Hodge structures. The adaptation of the calculations there extending the methods in St2] to the several variable log-geometry setting that corresponds to our situation will then give the result. As we have no substantive content to add to what is implicit in [Fu1, Fu2 and [St2 here we will not write out the details, but rather defer them to a later work.

An outstanding issue, as noted in [Fu1, Fu2, for a family (III.7), is in what way the mixed Hodge structure constructed in [Fu1, Fu2] using $\Omega_{X / S}^{\bullet}(\log X) \otimes \mathcal{O}_{X}$ relates to the limiting mixed Hodge structure given along a disc $\Delta_{\lambda}$ in $S=\Delta_{1} \times$ $\cdots \times \Delta_{\ell}$ in CKS1. One main point may be (III.5). In case $X$ is a local normal crossing variety, we have noted that $X_{\Delta_{\lambda}}$ is smooth and $X_{\Delta_{\lambda}} \rightarrow \Delta_{\lambda}$ is a standard family, so the result that the limiting mixed Hodge structures are the same is true in this case. Another outstanding matter is the construction of the monodromy logarithms $N_{i}$ from the $d t_{i} / t_{i}$ in the complexes constructed in Fu1, Fu2, and then to show that these give the structure as in CKS1. This also will be taken up in a later work.

Another issue, one that arises already when $X$ is a normal crossing variety whose singular locus $D=\cup D_{\alpha}$ has connected components $D_{\alpha}$, is this: In the exact sequence (II.1) when the mapping

$$
\oplus H^{0}\left(\mathcal{O}_{D_{\alpha}}\right) \stackrel{\delta}{\rightarrow} H^{2}\left(\operatorname{Ext}_{\mathcal{O}_{X}}^{0}\left(\Omega_{X}^{1}, \mathcal{O}_{X}\right)\right)
$$

is non-zero, the Kuranishi space may be unobstructed but the $D_{\alpha}$ cannot be individually smoothed 23 Suppose for example that there are three components so that projectively

$$
\mathbb{P}\left(\oplus H^{0}\left(\mathcal{O}_{D_{\alpha}}\right)\right)=\mathbb{P}^{2}
$$

\footnotetext{
${ }^{23}$ In the logarithmic deformation theoretic context, there are non-zero obstructions in the logarithmic analogue of $T_{X} \operatorname{Def}(X)$.
} 
pictured as

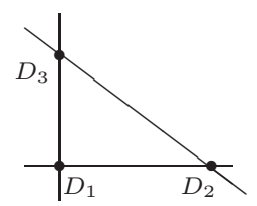

where the vertices correspond to the $H^{0}\left(\mathcal{O}_{D_{\alpha}}\right)$. If $\operatorname{dim}(\operatorname{ker} \delta)=2$, there are the following possibilities for the dotted line $L=\mathbb{P}(\operatorname{ker} \delta)$ :

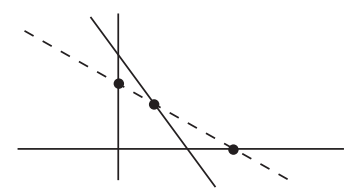

(ii)

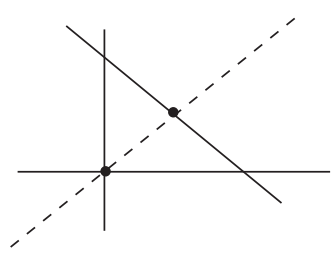

(iii)

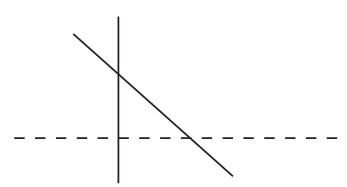

Here, (iii) does not occur since we are assuming that $X$ may be smoothed. For (ii), assuming that $T_{X} \operatorname{Def}(X)$ is unobstructed we have a 2-parameter family $X \rightarrow \Delta \times \Delta$ where along one axis $D_{1}$ is smoothed while $D_{2}$ and $D_{3}$ deform equisingularly. Along the other axis a similar thing happens with the roles of $D_{1}$ and $D_{2}, D_{3}$ interchanged.

In case (i) we have a 2-parameter family with three axes along each of which one pair from $D_{1}, D_{2}, D_{3}$ deforms equisingularly while the remaining component of $D$ is smoothed. Thus the picture of the tangent space to the 2-parameter family $X \rightarrow S$ is

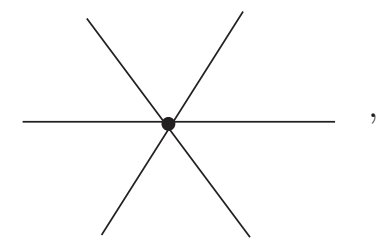

and for the family $X^{*} \rightarrow S^{*} \cong \Delta \times \Delta \backslash\{3$ lines $\}$ where the fibres are smooth we have

$$
\pi_{1}\left(S^{*}\right) \cong \pi_{1}\left(\mathbb{P}^{1} \backslash\{0,1, \infty\}\right)
$$


Thus to arrive at a CKS situation we have to blow up $S$ at the origin and resolve singularities to arrive at a standard situation $\widetilde{X} \rightarrow \widetilde{S}$ where locally $\widetilde{S}$ is a $\Delta \times \Delta$ with singular fibres over the two axes. The fibre $\widetilde{X}$ over the origin is related to $X$ in a standard way; it will have as one component a branched covering of the desingularization of $X$ along two of the $D_{\alpha}$, and the other components are easily described (if $X_{3}=\emptyset$ they are the projectivized normal bundles of the $D_{\alpha}$ in $X$ ). We are now back in the situation of CKS but with a different $X$. As in [KN for interesting examples this complexity does not arise, and for theoretical purposes we can at least begin by assuming that $\delta=0$ in (II.1).

\section{Proof of Theorem II}

We first will consider the question, informally stated as

$$
\text { What do we mean by } T(L M H S) \text { ? }
$$

Here, "LMHS" is the set of limiting mixed Hodge structures with monodromy $N$. Setting

$$
D_{N}=D \cup B(N),
$$

in [KU] there is defined on $D_{N}$ the structure of a "log-analytic varity with slits." In particular, the tangent space $T_{[F \bullet]} D_{N}$ to the underlying analytic variety at a point $\left[V, W_{\bullet}(N), F^{\bullet}\right]$ is defined, where the brackets denote the equivalence class of nilpotent orbits under the equivalence relation $F^{\bullet} \sim \exp (z N) \cdot F^{\bullet}$. This information may be refined if we do not pass to equivalence classes. Thus we define

$$
\widetilde{D}_{N}=D \cup \widetilde{B}(N)
$$

and seek to define $T_{F} \bullet \widetilde{D}_{N}$. For the subspace $T_{F}^{\bullet} \widetilde{B}(N)$ of $T_{F}^{\bullet} \widetilde{D}_{N}$, recalling that $\widetilde{D}_{N} \subset \check{D}$ we set

$$
T_{F} \bullet \widetilde{D}_{N}=\left\{\tau \in T_{F} \bullet \check{D}: \tau\left(F^{p}\right) \subset F^{p-1}\right\}=T_{F} \bullet \check{D} 24
$$

We next consider the question

\section{What is the algebro-geometric analogue of (IV.1)?}

This means: What algebro-geometric object maps to (IV.1), extending what is given for a smooth $X$ by (I.1)?

For this we recall that associated to a pair $(X, \xi)$, where $X$ is locally a product of normal crossing varieties and $\xi \in T_{X}^{0} \operatorname{Def}(X)$, is a limiting mixed Hodge structure whose underlying vector space is $\mathbb{H}^{m}\left(\Omega_{\mathcal{X}_{\xi} / \Delta_{\epsilon}}(\log X) \otimes \mathcal{O}_{X}\right)$. With the identification $T_{X} \operatorname{Def}(X)=\mathbb{E x t}_{\mathcal{O}_{X}}^{1}\left(\Omega_{X}^{1}, \mathcal{O}_{X}\right)$ in mind, we define

$$
T_{(X, \xi)} \operatorname{Def}(X, \xi)=\mathbb{E x t}_{\mathcal{O}_{X}}^{1}\left(\Omega_{X_{\xi} / \Delta_{\epsilon}}^{1}(\log X) \otimes \mathcal{O}_{X}, \mathcal{O}_{X}\right)
$$

where $\operatorname{Def}(X, \xi)$ is the set of deformations of the pair $(X, \xi) 25$ We shall not attempt here to give a precise definition of $\operatorname{Def}(X, \xi)$, but rather shall simply take IV.2

\footnotetext{
${ }^{24}$ Essentially we are interpreting the additional infinitesimal information that is present if we consider $D_{N}$ as a log-analytic variety.

${ }^{25}$ As was noted in the introduction, the right-hand side of IV.2 appears naturally in logarithmic deformation theory.
} 
as the definition of its tangent space. As partial justification, we observe that with this definition the obvious map

$$
\operatorname{Def}(X, \xi) \rightarrow \operatorname{Def}(X)
$$

together with the map $\Omega_{X}^{1} \rightarrow \Omega_{X_{\xi} / \Delta_{\epsilon}}^{1}(\log X)$, give

$$
\begin{array}{ccc}
T_{(X, \xi)} \operatorname{Def}(X, \xi) & \longrightarrow & T_{X} \operatorname{Def}(X) \\
\| & & \| \\
\mathbb{E x t}_{\mathcal{O}_{X}}^{1}\left(\Omega_{X_{\xi} / \Delta_{\epsilon}}^{1}(\log X) \otimes \mathcal{O}_{X}, \mathcal{O}_{X}\right) & \longrightarrow & \mathbb{E x t}_{\mathcal{O}_{X}}^{1}\left(\Omega_{X}^{1}, \mathcal{O}_{X}\right) .
\end{array}
$$

Turning to the definition of the maps in Theorem $\amalg$ from the middle row in the basic diagram (III.3) one may in the usual way infer the exact sequence of complexes

$$
0 \rightarrow \Omega_{X_{\xi} / \Delta_{\epsilon}}^{\bullet-1}(\log X) \otimes \mathcal{O}_{X} \rightarrow \Omega_{X_{\xi}}^{\bullet}(\log X) \otimes \mathcal{O}_{X} \rightarrow \Omega_{X_{\xi} / \Delta_{\epsilon}}^{\bullet}(\log X) \otimes \mathcal{O}_{X} \rightarrow 0
$$

The connecting homomorphism in the long exact hypercohomology sequence induces

$$
\mathbb{H}^{m}\left(\Omega_{X_{\xi} / \Delta_{\epsilon}}^{\bullet}(\log X) \otimes \mathcal{O}_{X}\right) \stackrel{\nabla_{\xi}}{\longrightarrow} \mathbb{H}^{m+1}\left(\Omega_{X_{\xi / \Delta_{\epsilon}}^{\bullet-1}}(\log X) \otimes \mathcal{O}_{X}\right)
$$

which satisfies

$$
\nabla_{\xi} F_{\xi}^{p} \subset F_{\xi}^{p-1}
$$

Then from (I.5) the element $\xi^{(1)} \in T_{(X, \xi)} \operatorname{Def}(X, \xi)$ gives an extension class in the above exact sequence of complexes, and using the identification

$$
\mathbb{H}^{m+1}\left(\Omega_{X_{\xi / \Delta_{\epsilon}}^{\bullet-1}}(\log X) \otimes \mathcal{O}_{X}\right)=\mathbb{H}^{m}\left(\Omega_{\mathfrak{X}_{\xi} / \Delta_{\epsilon}}^{\bullet}(\log X) \otimes \mathcal{O}_{X}\right)
$$

we obtain the map in the statement of Theorem [1] The fact that we map to End $_{\text {LMHS }}$ is a consequence of the naturality of the construction of the limiting mixed Hodge structure.

The geometric picture to keep in mind is this: The kernel of the map (II.1)

$$
\operatorname{Ext}_{\mathcal{O}_{X}}^{1}\left(\Omega_{X}^{1}, \mathcal{O}_{X}\right) \rightarrow H^{0}\left(\operatorname{Ext}_{\mathcal{O}_{X}}^{1}\left(\Omega_{X}^{1}, \mathcal{O}_{X}\right)\right)
$$

represents the tangents to the equisingular deformations of $X 26$ Modulo this kernel, the image of the above map reflects how the singularities are deforming. Given a smoothing deformation $\xi$, we may think of $\xi^{(1)}$ as giving us the infinitesimal change in this picture 27 We will now illustrate this by example where it will be quite clear how the map in the statement of Theorem $\amalg$ gives information beyond that in the differential

$$
T_{s_{0}} S \rightarrow T_{[F \bullet} D_{N}
$$

Here we are imagining a family $x \rightarrow S$ where $S=\Delta^{\ell}$ and where the fibres are smooth over $S^{*}=\Delta^{* \ell}$ with commuting monodromy logarithm transformations $N_{1}, \ldots, N_{\ell}$ around the axes. The corresponding nilpotent orbit is $\exp \left(z_{1} N_{1}+\right.$ $\left.\cdots z_{\ell} N_{\ell}\right) \cdot F^{\bullet}$.

\footnotetext{
${ }^{26}$ Recall that we are assuming that under any non-smoothing deformation $X^{\prime}$ of $X$, including an equisingular one, the deformed $X^{\prime}$ remains smoothable; the condition for this is in [Fr2].

${ }^{27}$ Of course, there is more information than this in $\xi$.
} 
Example: This will be a simpler version of the example from the beginning of Section VI, and we will use the notations from there. Then $F^{\bullet}$ is a single $F$ given by the span of the columns in the matrices below:

$$
\begin{aligned}
F \longleftrightarrow\left(\begin{array}{ccc}
1 & & \\
& 1 & \\
& & 1 \\
a_{11} & a_{12} & b_{1} \\
a_{21} & a_{22} & b_{2} \\
b_{1} & b_{2} & c
\end{array}\right), \quad a_{12}=a_{21} \\
{[F] \longleftrightarrow\left(\begin{array}{ccc}
1 & & \\
& 1 & \\
0 & a_{12} & b_{1} \\
a_{21} & 0 & b_{2} \\
b_{1} & b_{2} & c
\end{array}\right) }
\end{aligned}
$$

where the notation $\longleftrightarrow$ means "corresponds to." For $[F]$ we have normalized the point on the several variable nilpotent orbit by $a_{11}=a_{22}=0$ :

$$
\begin{array}{r}
T_{F} \check{D} \longleftrightarrow\left(\begin{array}{ccc}
d a_{11} & d a_{12} & d b_{1} \\
d a_{21} & d a_{22} & d b_{2} \\
d b_{1} & d b_{2} & d c
\end{array}\right), \\
T_{[F]} D_{N} \longleftrightarrow\left(\begin{array}{ccc}
0 & d a_{12} & d b_{1} \\
d a_{21} & 0 & d b_{2} \\
d b_{1} & d b_{2} & d c
\end{array}\right) .
\end{array}
$$

Geometrically, the second contains the information in $T_{X} \mathcal{C} \subset T_{X} \partial \mathcal{M}_{3}$, while the first contains this information plus the information in the normal space to $\mathcal{C}$ in $\overline{\mathcal{M}}_{3}$; i.e., the refined direction of approach to $X$ in the boundary of $\mathcal{M}_{3}$. Here the term "refined direction of approach" means the following: The crude normal direction of approach to $X$ is given by $N_{1}, N_{2}$, which may be thought of as the normal direction of approach to the image of $X$ in $D_{N}$. The refined direction of approach picks out more subtle information beyond that given simply by the logarithmic terms in the period matrix.

Still referring to the next section for the notations, the $N$-strings associated to the limiting mixed Hodge structure in this example may be written as

$$
\begin{gathered}
H^{0}(-1) \rightarrow H^{0} \\
H^{1} .
\end{gathered}
$$

The extension data in $\operatorname{Ext}_{\mathrm{MHS}}^{1}\left(\mathrm{Gr}_{1}, G_{0}\right)=\operatorname{Ext}_{\mathrm{MHS}}^{1}\left(H^{1}(\widetilde{X}), H^{0}(D)\right)$ corresponds to $b_{1}, b_{2}$, while that for $\operatorname{Ext}_{\mathrm{MHS}}^{1}\left(H^{0}(D)(-1), H^{0}(D)\right)$ corresponds to the $2 \times 2$ symmetric matrix $\left(a_{i j}\right)$. In this case only the off-diagonal terms are invariant under $F^{\bullet} \rightarrow \exp \left(z_{1} N_{1}+z_{2} N_{2}\right) \cdot F^{\bullet}$, while the diagonal terms require the choice of $\xi$. 
From a cohomological perspective, the $F$ in the limiting mixed Hodge structure is

$$
H^{0}\left(\Omega_{\widetilde{X}}^{1}(\log (p+q))\right)
$$

and the matrix $d F$ in (IV.4) is in

$$
\operatorname{Hom}_{s}\left(F, \mathbb{C}^{4} / F\right) \cong \operatorname{Hom}_{s}\left(H^{0}\left(\Omega_{\widetilde{X}}^{1}(\log (p+q)), H^{1}\left(\mathcal{O}_{X}\right)\right)\right) 28
$$

where $\operatorname{Hom}_{s}$ are the symmetric maps. Under the inclusion

$$
H^{0}\left(\Omega_{\widetilde{X}}^{1}\right) \hookrightarrow H^{0}\left(\Omega_{X}^{1}(\log (p+q))\right)
$$

the matrix (IV.5) contains a part in $\operatorname{Hom}\left(H^{0}\left(\Omega_{\widetilde{X}}^{1}\right), H^{1}\left(\mathcal{O}_{X}\right)\right)$. The term $d c$ is in

$$
\operatorname{Hom}\left(H^{0}\left(\Omega_{\widetilde{X}}^{1}\right), H^{1}\left(\mathcal{O}_{\tilde{X}}\right)\right)
$$

and $\left(d b_{1}, d b_{2}\right)$ belongs to

$$
\operatorname{Hom}\left(H^{0}\left(\Omega_{\widetilde{X}}^{1}\right), H^{1}\left(\mathcal{O}_{X}\right) / H^{1}\left(\mathcal{O}_{\tilde{X}}\right)\right) .
$$

An extreme example of the extra information is given by the genus 2 curve degenerations

(i)

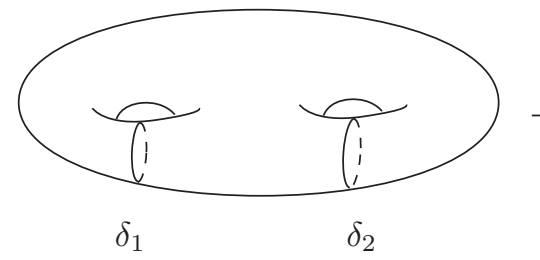

(ii)

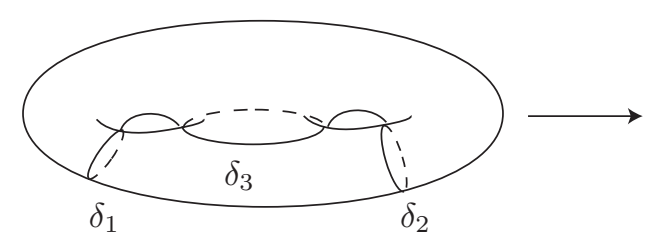

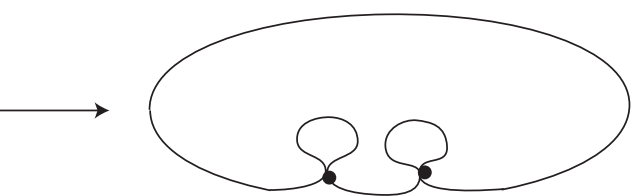

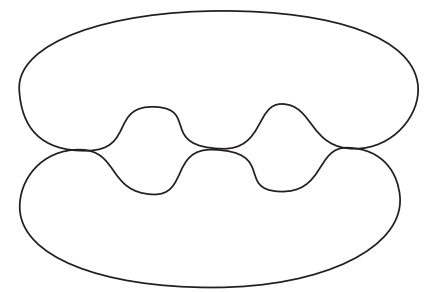

In each case the polarized limiting mixed Hodge structure is

$$
\stackrel{2}{\oplus} \mathbb{Q}(-1) \rightarrow \stackrel{2}{\oplus} \mathbb{Q}
$$

\footnotetext{
${ }^{28}$ Here we are identifying $H^{1}\left(\mathcal{O}_{X}\right)$ with $V_{\xi / F}$ where $\left(V_{\xi}, W \bullet(N), F\right)$ is the limiting mixed Hodge structure with $N=N_{1}+N_{2}$.
} 
and the $\operatorname{Ext}_{\text {PLMS }}^{1}(\bullet, \bullet)$ is given by a $2 \times 2$ symmetric case. For $T_{F} \bullet \widetilde{D}_{N}$ and $T_{[F} \bullet D_{N}$ they are

$$
\begin{array}{lll}
T_{F} \bullet \check{D}_{N} & \left(\begin{array}{ll}
a & b \\
b & a
\end{array}\right) & \left(\begin{array}{ll}
a & b \\
b & a
\end{array}\right) \\
T_{[F \bullet]} D_{N} & \left(\begin{array}{ll}
0 & b \\
b & 0
\end{array}\right) & \left(\begin{array}{ll}
0 & 0 \\
0 & 0
\end{array}\right)
\end{array}
$$

This illustrates the additional information contained in considering the map $T_{(X, \xi)} \operatorname{Def}(X, \xi) \rightarrow$ $T_{F} \cdot \widetilde{D}_{N}$.

The above examples are of course special. However, the regularization of logarithmic integrals phenomenon they illustrate are fairly general. For instance, in GGR the generic degenerations of Hodge structures of odd weight $n=2 m+1$ are given, for $1 \leqq k \leqq n$, by a specialization

$$
X_{t} \rightarrow X_{0}
$$

where locally in $\mathbb{C}^{2 m+2} X_{0}$ is given by

$$
x_{1} x_{2}+\cdots+x_{2 k-1} x_{2 k}=0
$$

and thus has a double locus of codimension $k$. For $k=1$ we have that $X_{0}=X$ has a codimension one double $2 m$-fold $X_{\text {sing }}=X_{1}$. For $k=m, X_{0}$ has an ordinary isolated quadratic singularity. For $k \geqq 2$ we have to blow up $X_{0}$ to achieve a standard family. The limiting mixed Hodge structures are

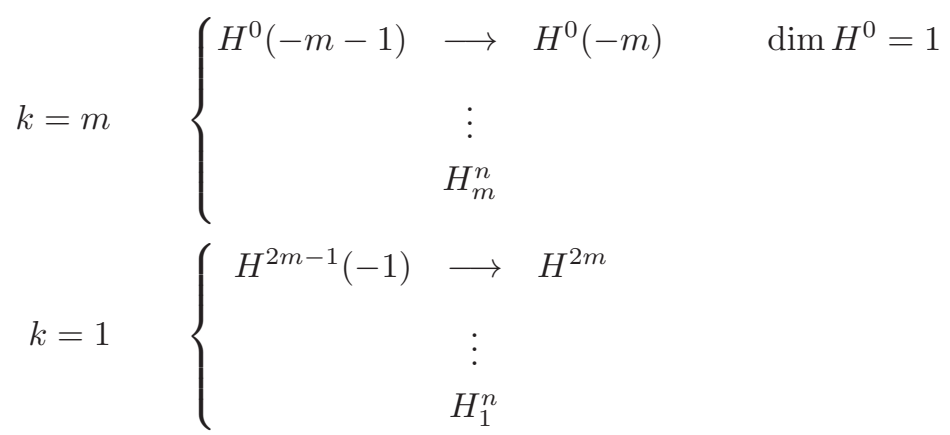

For $H_{m}^{n}$ the Hodge numbers $h_{k}^{p, q}$ are the same as the $h^{p, q}$ for the original polarized Hodge structure on $H^{n}\left(X_{t}\right)$, except that

$$
h_{k}^{m+k, m-k+1}=h^{m+k, m-k+1}-1 .
$$

Geometrically we have a class $\omega_{t}$ in $H^{0}\left(\Omega_{X_{t}}^{n}\right)$ that acquires a pole of order $k$ along $X_{\text {sing }}$, and by a residue-type construction we end up with a class in $H^{n-m}\left(\Omega_{X^{[m]}}^{m-k+1}\right)$. The above analysis of regularizing an integral

$$
\lim _{t \rightarrow 0} \int_{\gamma_{t}} \omega_{t}
$$

then will carry over. The details of this will be carried out in a future work.

Finally we would like to give a general cohomological description of the extra information in the map in Theorem $\amalg$. We will do this in case $X$ is a nodal curve; 
this description will extend to the general case when $X$ has only an ordinary double locus $D=X_{\text {sing. }}$. With this assumption the right-hand column in the basic diagram (III.3) gives a map

$$
\Omega_{X_{\xi} / \Delta_{\epsilon}}^{1}(\log X) \otimes \mathcal{O}_{X} \rightarrow \mathcal{O}_{D}
$$

which induces a map

$$
\mathbb{E x t}_{\mathcal{O}_{X}}^{1}\left(\mathcal{O}_{D}, \mathcal{O}_{X}\right) \rightarrow \mathbb{E x t}_{\mathcal{O}_{X}}^{1}\left(\Omega_{X_{\xi} / \Delta_{\epsilon}}^{1}(\log X) \otimes \mathcal{O}_{X}, \mathcal{O}_{X}\right) .
$$

The image of this map represents the "extra information" contribution to the map in Theorem II The local result we need to describe this for $X$ a germ given by $x y=0$ is

$$
\begin{gathered}
\text { Ext }_{\mathcal{O}_{X}}^{1}\left(\mathcal{O}_{D}, \mathcal{O}_{X}\right) \cong \mathcal{O}_{D} \text { and is generated by the extension } \\
0 \rightarrow \mathcal{O}_{X} \rightarrow\left(a_{1}\right)_{*} \mathcal{O}_{X^{[1]}} \rightarrow \mathcal{O}_{D} \rightarrow 0 .
\end{gathered}
$$

In the case under consideration,

$$
\mathbb{E x t}_{\mathcal{O}_{X}}^{1}\left(\mathcal{O}_{D}, \mathcal{O}_{X}\right) \cong H^{0}\left(\operatorname{Ext}_{\mathcal{O}_{X}}^{1}\left(\mathcal{O}_{D}, \mathcal{O}_{X}\right)\right)
$$

and there are the number of connected components of $D$ additional parameters picked up in the additional information.

A cohomological formulation that identifies the $N$ in a limiting mixed Hodge structure is this. Recall the bottom two rows in the basic diagram (III.3), where if we use the notation $\mathcal{S}=\operatorname{ker}\left\{\left(a_{2}\right)_{*} \mathcal{O}_{X}{ }^{[2]} \rightarrow\left(a_{3}\right)_{*} \mathcal{O}_{X^{[3]}}\right\}$ and identify $\pi^{*} \Omega_{\Delta_{\epsilon}}^{1}(\log 0) \otimes$ $\mathcal{O}_{X}$ with $\mathcal{O}_{X}$ we have

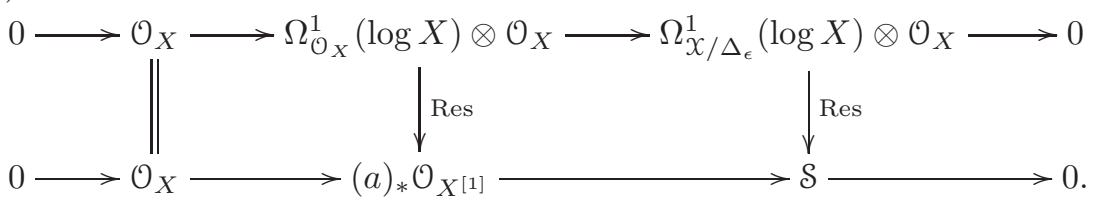

This gives

$$
\mathbb{E x t}_{\mathcal{O}_{X}}^{1}\left(\mathcal{S}, \mathcal{O}_{X}\right) \stackrel{\text { Res }^{*}}{\longrightarrow} \mathbb{E x t}_{\mathcal{O}_{X}}^{1}\left(\Omega_{X / \Delta_{\epsilon}}^{1}(\log X) \otimes \mathcal{O}_{X}, \mathcal{O}_{X}\right) \rightarrow F^{-1} \operatorname{End}_{\text {LMHS }}
$$

Then by interpreting the construction in [St1] we find that

the image of the extension class in the bottom row of (IV.8) is the monodromy logarithm $N$.

Finally, we would like to point out the paper $\mathrm{Ca}-\mathrm{Fe}$ in which the notion of an infinitesimal variation of Hodge structure at infinity is defined. Their definition pertains to equivalence classes of limiting mixed Hodge structures for several variable nilpotent orbits as in CKS1. In the above example the definition in $\mathrm{Ca}-\mathrm{Fe}$ would record the data

$$
\left\{N_{1}, N_{2}: d a_{12}, d b_{1}, d b_{2}, d c\right\} .
$$

Roughly speaking, this data corresponds to $T B(N)$ and to the normal space to $B(N)$ in $D_{N}$, while that in Theorem $\amalg$ may be thought of as having the information in some sort of blow up of the normal space to $B(N)$ in $D_{N}$. 


\section{Proof of Theorem III}

The proof of Theorem III will be given following several preliminary discussions on the following topics:

- nilpotent orbits and the reduced limit period mapping;

- monodromy cone structure associated to a normal crossing variety;

- the differential of the reduced limit period mapping.

Nilpotent orbits and the reduced limit period mapping. We begin by recalling some definitions and results from [CKS1, [KP1, [KP2], GGK], GG] and GGR, the last two of whose notations we shall generally follow. We let

- $\widetilde{B}(N)=$ set of nilpotent orbits $\left(F^{\bullet}, N\right)$.

Here, $D=G_{\mathbb{R}} / H$ is a Mumford-Tate domain embedded as an open $G_{\mathbb{R}}$-orbit in its compact dual $\check{D}=G_{\mathbb{C}} / P$. The Mumford-Tate domain structure on $D$ gives a realization of $\check{D}$ as a set of filtrations $F^{\bullet}=\left\{F^{m} \subset F^{m-1} \subset \cdots \subset F^{0}=V_{\mathbb{C}}\right\}$ on the complexification of a $\mathbb{Q}$-vector space $V$. The monodromy logarithm $N \in \mathfrak{g}^{\text {nilp }} \subset$ $\operatorname{End}(V)$ is a nilpotent endomorphism of $V$ that gives rise to the monodromy weight filtration, which we center at zero,

$$
W_{-k}(N) \subset \cdots \subset W_{0}(N) \subset \cdots \subset W_{k}(N)=V, \quad k \leqq m
$$

where $N^{k+1}=0, N^{k} \neq 0(k \leqq m)$. The conditions to be a nilpotent orbit are

(i) $N F^{p} \subset F^{p-1}$;

(ii) $\exp (z N) \cdot F^{\bullet} \in D$ for $\operatorname{Im} z \gg 0$.

It is known and of central importance that ([CKS1]

(V.1) $\left(F^{\bullet}, N\right)$ is a nilpotent orbit

$\Longleftrightarrow\left(V, W_{\bullet}(N), F^{\bullet}\right)$ is a limiting mixed Hodge structure .

Here we recall that a limiting mixed Hodge structure $\left(V, W_{\bullet}(N), F^{\bullet}\right)$ is given by $F^{\bullet}$ and $N$ where $W_{\bullet}(N)$ is the monodromy weight filtration and where $F^{\bullet}$ reduces on $\mathrm{Gr}_{k}^{W \cdot(N)}$ a pure Hodge structure of weight $k$. All of our limiting mixed Hodge structures will be polarized by a $Q: V \otimes V \rightarrow \mathbb{Q}$ (cf. [Sc] and [CKS1]).

Two nilpotent orbits $\left(N, F^{\bullet}\right)$ and $\left(N, F^{\bullet}\right)$ are said to be equivalent if

$$
F^{\prime \bullet}=\exp (z N) \cdot F^{\bullet}
$$

for some $z \in \mathbb{C}$; i.e., if they lie in the same $\exp (\mathbb{C} N)$ orbit in $\check{D}$. We let

- $B(N)=\exp (\mathbb{C} N) \backslash \widetilde{B}(N)=$ set of nilpotent orbits modulo equivalence.

Assuming that $N \neq 0$ there is a reduced limit period mapping (called a naïve limit in KP1]

$$
\Phi_{\infty}: B(N) \rightarrow \partial D
$$

whose image lies in a $G_{\mathbb{R}^{-}}$orbit. The definition is

$$
\Phi_{\infty}\left(F^{\bullet}, N\right)=\lim _{z \rightarrow \infty} \exp (z N) \cdot F^{\bullet}=: F_{\infty}^{\bullet} .
$$

If we think of $\check{D}$ as embedded in a product of projective spaces via the Plücker embeddings of the individual subspaces $F^{p} \subset V_{\mathbb{C}}$, then since $N$ is nilpotent the Plücker coordinates of $\exp (z N) \cdot F^{p}$ are polynomials in $z$ and thus have a welldefined limit at $z=\infty$. In effect $\Phi_{\infty}\left(F^{\bullet}, N\right)$ picks out the highest powers of $z$ in the Plücker coordinates of $\exp (z N) F^{p}$. An elementary general fact is that for any 
nilpotent $N$ the vector field on $\check{D}$ induced by the action of the 1-parameter group $\exp (z N)$ vanishes to $2^{\text {nd }}$ order at the limit point $F_{\infty}^{\bullet}$, so that the reduced limit period mapping is well defined on the quotient space $B(N)$ of $\widetilde{B}(N)$.

One of the important features of the reduced limit period mapping is

(V.3) The mapping (V.2) factors

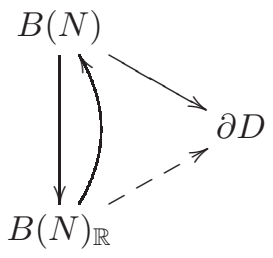

through the set $B(N)_{\mathbb{R}}$ of equivalence classes of $\mathbb{R}$-split limiting mixed Hodge structures.

- Associated to a mixed Hodge structure $\left(V, W_{\bullet}, F^{\bullet}\right)$ there is the canonical Deligne bigrading

$$
V_{\mathbb{C}}=\oplus I^{p, q}
$$

where

$$
\left\{\begin{array}{l}
F^{p}=\underset{r \geqq p}{\oplus} I^{r, \bullet} \\
W_{k}=\underset{p+q \leqq k}{\oplus} I^{p, q} \\
I^{p, q} \equiv \bar{I}^{q, p} \text { modulo } W_{p+q-2}
\end{array}\right.
$$

- The mixed Hodge structure is $\mathbb{R}$-split in case

$$
I^{p, q}=\overline{I^{q, p}}
$$

canonically associated to a mixed Hodge structure $\left(V, W_{\bullet}, F^{\bullet}\right)$ is an $\mathbb{R}$-split mixed Hodge structure $\left(V, W_{\bullet}, \widetilde{F}^{\bullet}\right)$;

- If $\left(V, W \bullet(N), F^{\bullet}\right)$ is a limiting mixed Hodge structure, then so is the $\mathbb{R}$-split mixed Hodge structure $\left(V, W \bullet(N), \widetilde{F}^{\bullet}\right)$, and conversely.

It follows from this last propery that we have the factorization (V.3). For the time being we will assume that

$$
\left(V, W_{\bullet}(N), F^{\bullet}\right) \text { is } \mathbb{R} \text {-split. }
$$

In this case the filtration $F_{\infty}^{\bullet}$ is related to $F^{\bullet}$ by

$$
F_{\infty}^{p}=\underset{q \leqq m-p}{\oplus} I^{\bullet, q},
$$

where $m$ is the weight of the Hodge structure under consideration.

- If $\left(V, W_{\bullet}, F^{\bullet}\right)$ is a mixed Hodge structure, then the inclusion $\mathfrak{g} \subset \operatorname{End}_{Q}(V, V)$ induces on $\mathfrak{g}$ a mixed Hodge structure $\left(\mathfrak{g}, W_{\bullet}, \mathfrak{g}, F_{\mathfrak{g}}^{\bullet}\right)$;

- Under the assumption (V.5) we have

$$
\mathfrak{g}_{\mathbb{C}}=\oplus I_{\mathfrak{g}}^{p, q}
$$

and

$$
F_{\mathfrak{g}, \infty}^{p}=\underset{q \leqq p}{\oplus} I_{\mathfrak{g}}^{\bullet, q}
$$


- the monodromy logarithm $N \in I_{\mathfrak{g}}^{-1,-1}$;

- with the identifications

$$
\left\{\begin{array}{l}
T_{F_{\mathfrak{g}}} \check{D}=\underset{p \leqq-1}{\oplus} I_{\mathfrak{g}}^{p, \bullet}=F_{\mathfrak{g}}^{-1} \\
T_{F_{\mathfrak{g}, \infty}} \check{D}=\underset{q \geqq 1}{\oplus} I_{\mathfrak{g}}^{\bullet, q}=F_{\mathfrak{g}, \infty}^{-1}
\end{array}\right.
$$

(V.7) the differential

$$
\Phi_{\infty, *}: T_{F} \cdot B(N)_{\mathbb{R}} \rightarrow T_{F_{\infty}} \check{D}
$$

of the reduced limit period mapping is the identity on $I_{\mathfrak{g}}^{p, q}$ for $q \geqq 1$ and is zero on $I_{\mathfrak{g}}^{p, q}$ for $q<0$.

Pictorially, we picture $I_{\mathfrak{g}}^{p, q}$ in the $(p, q)$ plane

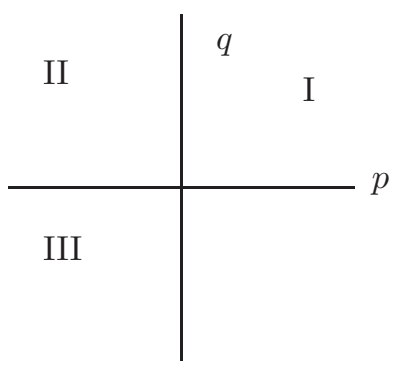

Then

$$
\begin{aligned}
T_{F} \cdot \widetilde{B}(N) & \subseteq \mathrm{I} \cup \mathrm{II} \\
T_{F_{\infty}} \check{D} & \cong \mathrm{II} \cup \mathrm{III}
\end{aligned}
$$

and $\Phi_{\infty, *}$ is the identity on the interior of II with

$$
\left\{\begin{aligned}
\operatorname{ker} \Phi_{\infty, *} & =\mathrm{I} \\
\operatorname{coker} \Phi_{\infty, *} & =\text { III. }
\end{aligned}\right.
$$

Monodromy cone structure associated to a normal crossing variety. More generally, associated to a nilpotent cone

$$
\sigma=\operatorname{span}_{\mathbb{Q} \geqq 0}\left\{N_{1}, \ldots, N_{\ell}\right\}
$$

with interior $\sigma^{\circ}$, from CKS1, CKS2 there is an intricate and deep structure of nilpotent orbits, or equivalently limiting mixed Hodge structures in several variables. Among the properties of this structure are

- the monodromy weight filtration is independent of $N \in \sigma^{0}$ ( $\underline{\mathrm{CK}}$ );

- the limiting mixed Hodge structure associated to a nilpotent orbit $\left(F^{\bullet}, N\right)$ is independent of $N \in \sigma^{\circ}$;

- denoting by $\Delta^{*}(r)$ a punctured disc of radius $r$, in the manner described in CKS1 on $\Delta^{*}\left(r_{1}\right) \times \cdots \times \Delta^{*}\left(r_{\ell}\right)$ there are several variable nilpotent orbits

$$
\exp \left(z_{1} N_{1}+\cdots+z_{\ell} N_{\ell}\right) \cdot F^{\bullet}, \quad \operatorname{Im} z_{i} \gg 0
$$

which induce variations of mixed Hodge structure ( $[\mathrm{St}-\mathrm{Zu}]$ ) on the axes in $\Delta\left(r_{1}\right) \times \cdots \times \Delta\left(r_{\ell}\right)$. 
An important example of this cone structure is provided by a normal crossing variety $X$ for which there exists a $\xi \in T_{X} \operatorname{Def}(X)$ such that

$$
\xi_{D_{a}} \neq 0 \text { for each of the connected components } D_{a}, a \in A \text {, of } D \text {. }
$$

We will describe $\sigma_{X}$ when the following special condition is satisfied:

$$
\begin{aligned}
& \text { for each } a \in A \text {, there is } \xi_{A} \in \mathbb{E x t}_{\mathcal{O}_{X}}^{1}\left(\Omega_{X}^{1}, \mathcal{O}_{X}\right) \text { such that } \\
& \xi_{A, a} \neq 0 \text { while } \xi_{A, b}=0 \text { for } b \notin A \text {. }
\end{aligned}
$$

Geometrically, to first order we may deform $X$ smoothing the component $D_{a}$ of $X_{\text {sing }}$ while remaining locally equisingular along the other components $D_{b}, b \neq a$. Under the assumption (V.9) we may to $1^{\text {st }}$ order independently smooth the components $D_{a}$ of the singular locus $D$ of $X$. Then there are monodromy transformations $N_{a}, a \in N$, that lead to a nilpotent cone.

In general the map

$$
\operatorname{Ext}_{\mathcal{O}_{X}}^{1}\left(\Omega_{X}^{1}, \mathcal{O}_{X}\right) \rightarrow \underset{a \in A}{\oplus} H^{0}\left(\mathcal{O}_{D_{a}}\right)
$$

will fail to be surjective and additional constructions are needed to obtain a set of monodromy cones described by the combinatorics of how the image of the mapping (V.10 meets the "coordinate axes" given by the right-hand term. The details of this will be given in the aforementioned work in progress. In that work we hope to also give the description of the cone in case $X$ is locally a product of normal crossing varieties. In this situation the faces of the cone will correspond to where factors in the local product description become smoothed, as well as to where components in the stratification of $X_{\text {sing }}$ become smoothed.

Proof of Theorem ЏI. The proof now follows from a very particular case of Robles' result $\mathrm{Ro}$. In this special case the argument is much simpler and goes as follows.

The first step is to identify the tangent spaces to the $G_{\mathbb{R}}$-orbit

$$
\mathcal{O}_{F_{\infty}}=: G_{\mathbb{R}} \cdot F_{\infty}^{\bullet} \subset \partial D .
$$

This is done in KP1 and later in GGK and GG; we shall follow the notations and indexing in the latter. From Section III.A we have for the real tangent space

$$
\begin{aligned}
T_{F_{\infty}}^{\mathbb{R}} \mathcal{O}_{F} \cdot & =\operatorname{Image}\left\{\mathfrak{g}_{\mathbb{R}} \rightarrow \mathfrak{g}_{\mathbb{C}} / F_{\infty}^{0} \mathfrak{g}_{\mathbb{C}}\right\} \\
& \cong \bigoplus_{\substack{q>0 \\
p \geqq 0}}\left(\mathfrak{g}^{p, q}+q^{q, p}\right) \cap \mathfrak{g}_{\mathbb{R}} \oplus \bigoplus_{q \geqq p>0}\left(\mathfrak{g}^{p, q} \oplus q^{q, p}\right) \cap \mathfrak{g}_{\mathbb{R}} \\
& \cong \operatorname{Res}_{\mathbb{C} / \mathbb{R}}\left\{\bigoplus_{\substack{q>0 \\
p \leqq 0}} \mathfrak{g}^{p, q}\right\} \oplus \bigoplus_{q \geqq p>0}\left(\mathfrak{g}^{p, q} \oplus q^{q, p}\right) \cap \mathfrak{g}_{\mathbb{R}}
\end{aligned}
$$

where $\operatorname{Res}_{\mathbb{C} / \mathbb{R}}$ is the restriction of scalars from $\mathbb{C}$ to $\mathbb{R}$ that maps a complex vector space to the same space now considered as a vector space over $\mathbb{C} \subset \mathbb{R}$. From the discussion above we see that $T_{(X, \xi)} \operatorname{Def}(X, \xi)$ maps to the first factor, which is in the tangent space to the $G_{\mathbb{R}}$-orbit $\mathcal{O}_{F_{\infty}}$.

As noted above, what one would like is to show that the interiors of the faces of

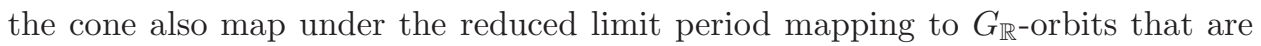
in the closure of the image of $\sigma_{X}$. 


\section{The hierarchy of MiXed Hodge Structures}

In this discussion we will restrict to a standard family $x \rightarrow \Delta$. To this situation there are naturally associated four mixed Hodge structures:

(i) the mixed Hodge structure on $H^{m}(X)$;

(ii) that part of the limiting mixed Hodge structure that can be constructed from $X$ alone;

(iii) the limiting mixed Hodge structure associated to $X \rightarrow \Delta$, modulo the equivalence $F^{\bullet} \sim \exp (z N) \cdot F^{\bullet}$ arising from a change of parameter on $\Delta 29$

(iv) the limiting mixed Hodge structure associated to the pair $(X, \xi)$ where $\xi \in T_{X} \operatorname{Def}(X)$.

We recall from the introduction the

THEOREM IV: In a manner to be explained in the proof, there are strict implications

$$
\text { (iv) } \Longrightarrow \text { (iii) } \Longrightarrow \text { (ii) } \Longrightarrow \text { (i). }
$$

Intuitively there is successively strictly less information in the data arising from the situations (iv), (iii), (ii), (i). Before turning to the proof we will illustrate the result in the following

Example 30 Suppose that $X_{t}$ is a smooth curve of genus $g=3$ whose limit $X$ is an irreducible nodal curve whose normalization $\widetilde{X}$ has genus $\widetilde{g}=1$

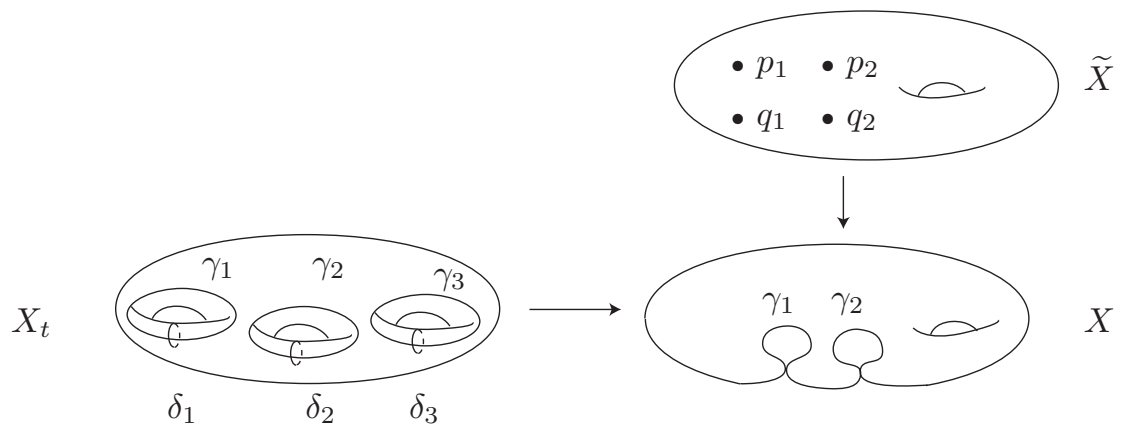

Setting $\ell(t)=(1 / 2 \pi i) \log t$ and using the symplectic basis drawn above for $H_{1}\left(X_{t}, \mathbb{Z}\right)$, the normalized period matrix is

$$
\left(\begin{array}{ccc}
1 & & \\
& 1 & 1 \\
\ell(t)+a_{11}(t) & a_{12}(t) & b_{1}(t) \\
a_{21}(t) & \ell(t)+a_{22}(t) & b_{2}(t) \\
b_{1}(t) & b_{2}(t) & c(t)
\end{array}\right)
$$

\footnotetext{
${ }^{29}$ We have seen that this data will depend only on the $1^{\text {st }}$ order neighborhood of $X$ in $X$.

${ }^{30}$ This is an extension to $g=3$ of the case $g=2$ in $\mathrm{Ca}$.
} 
where the $a_{i j}(t)=a_{j i}(t), b_{i}(t)$ and $c(t)$ are holomorphic in the disc and $\operatorname{Im} c(t)>0$. With the choice $t$ of parameter the nilpotent orbit is

$$
\left(\begin{array}{ccc}
1 & & \\
& 1 & 1 \\
\ell(t)+a_{11} & a_{12} & b_{2} \\
a_{21} & \ell(t)+a_{22} & b_{2} \\
b_{1} & b_{2} & c
\end{array}\right)
$$

where $a_{i j}=a_{i j}(0), b_{i}=b_{i}(0)$ and $c=c(0)$. Letting $\omega_{i}(t)$ be the holomorphic differentials on $X$ with limits $\omega_{i}$ on $X$ that pull up to $\widetilde{\omega}_{i}$ on $\widetilde{X}$, we have

- $\widetilde{\omega}_{1}, \widetilde{\omega}_{2}$ are differentials of the $3^{\text {rd }}$ kind on $\widetilde{X}$ with divisor $p_{i}+q_{i}$ and $\operatorname{Res}_{p_{i}} \widetilde{\omega}_{i}=+1, \operatorname{Res}_{q_{i}} \widetilde{\omega}_{i}=-1$ for $i=1,2$;

- $\widetilde{\omega}_{3}$ is a holomorphic differential on $\widetilde{X}$.

Under a reparamatrization $t^{\prime}=e^{2 \pi i \lambda} t$,

$$
a_{i i}\left(t^{\prime}\right)=a_{i i}(t)+\lambda
$$

and all other entries in the period matrix evaluated at $t=0$ are unchanged.

We note that implicit in the choice of symplectic basis is the monodromy weight filtration

$$
\left\{\begin{array}{r}
W_{0}=\operatorname{span}\left\{\delta_{1}, \delta_{2}\right\} \\
W_{2} / W_{1} \cong \operatorname{span}\left\{\gamma_{1}, \gamma_{2}\right\} \\
W_{1} / W_{0} \cong \operatorname{span}\left\{\delta_{3}, \gamma_{3}\right\} .
\end{array}\right.
$$

The entries in the above period matrix at $t=0$ are (cf. $\mathrm{Ca}$ )

$\left(I_{1}\right) c$ is the period of the elliptic curve $\tilde{X}$;

$\left(I_{2}\right) b_{i}$ is the image of $\operatorname{AJ}_{\widetilde{X}}\left(p_{i}-q_{i}\right)$ in $J(\widetilde{X})$; this gives the extension data in

$$
0 \rightarrow \mathrm{Gr}_{0} \rightarrow \mathrm{Gr}_{1} \rightarrow \mathrm{Gr}_{1} / \mathrm{Gr}_{0} \rightarrow 0
$$

as described in $\mathrm{Ca}$;

$\left(I_{3}\right)$ with suitable normalization of the $\widetilde{\omega}_{i}$,

$$
a_{i j}=\int_{q_{i}}^{p_{j}} \widetilde{\omega}_{i}, \quad i \neq j
$$

$\left(I_{4}\right)$ finally, with a choice of parameter $t$ we may uniquely define the improper integrals

$$
\int_{q_{i}}^{p_{i}} \widetilde{\omega}_{i}
$$

This means that if $\gamma_{i, t}$ is the above curve on $X_{t}$ for $t \neq 0$

$$
\int_{\gamma_{i, t}} \omega_{i, t}=\ell(t)+a_{i i}(t),
$$

and then on $\widetilde{X}$ we will have

$$
\lim _{\substack{p_{i}^{\prime} \rightarrow p_{i} \\ q_{i}^{\prime} \rightarrow q_{i}}} \int_{q_{i}^{\prime}}^{p_{i}^{\prime}} \widetilde{\omega}_{i}=a_{i i}(0)
$$


where the picture is

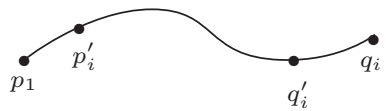

and where the logarithmic singularities at the endpoints cancel.

The $a_{i j}$ for $i \neq j$ record the part of the "extension upon extension" data in $\mathrm{Gr}_{2} / G_{1}$ over $\mathrm{Gr}_{1} / \mathrm{Gr}_{0}$ that is invariant under reparametrization, and the $a_{i i}$ record the full extension data.

Algebro-geometrically the picture is the following. Denoting by $\mathcal{M}_{g}$ the moduli space of the stable curves of genus $g$, and by $\overline{\mathcal{M}}_{g}$ the Deligne-Mumford compactification, the curve $X$ gives a point in

$$
\partial \mathcal{M}_{3} \subset \overline{\mathcal{M}}_{3} .
$$

More specifically, $X$ defines a point in a codimension- 1 component $\mathcal{C}$ of the stratified variety $\partial \mathcal{M}_{3}$. Then

- $\operatorname{dim} \mathcal{C}=4$ and $c, b_{1}, b_{2}$ and $a_{12}$ are local coordinates in $\mathcal{C}$;

- $a_{11}$ and $a_{22}$ give normal parameters to $\mathcal{C}$ in $\overline{\mathcal{M}}_{3}$.

The difference between (ii) and (iii) in this case is that (iii) contains the information in the weight filtration, which is information that is not obtainable from that on $X$ alone (see the subsequent discussion).

To explain (ii) we will picture a limiting mixed structure in terms of the $N$-strings as

$$
\begin{gathered}
H^{0}(-m) \rightarrow \cdots \quad \rightarrow H^{0}(-1) \stackrel{N}{\rightarrow} H^{0} \\
\vdots \\
H^{m-1}(-1) \rightarrow H^{m-1} \\
H^{m}
\end{gathered}
$$

where $H^{k}$ is a pure Hodge structure of weight $k$. It is this presentation that is especially useful in the computation of examples [GG] and GGR. Equivalent data to the above are the following parts of a polarized limiting mixed Hodge structure $\left(V, W \bullet(N), F^{\bullet}\right)$

- the Hodge structures $\mathrm{Gr}_{k}^{W \cdot(N)}$;

- the iterated $N$ operators on the $N$-strings.

It is known GG, $\mathrm{Ro}$ that this data always arises from a non-unique limiting mixed Hodge structure.

Our main result, Theorem $\nabla$ in the introduction, is that, under the assumption that $X$ is smoothable but with no $\xi \in T_{X}^{0} \operatorname{Def}(X)$ singled out, we may compute the $H^{m-i}(-j)$ 's above purely in terms of $X$ alone. For this we will use the maps

$$
\text { Rest : } H^{q}\left(X^{[k]}\right) \rightarrow H^{q}\left(X^{[k+1]}\right)
$$

obtained by the alternating sums of the restriction maps, and the suitably alternated Gysin maps

$$
\text { Gy }: H^{q}\left(X^{[k]}\right)(-1) \rightarrow H^{q+2}\left(X^{[k-1]}\right) \text {. }
$$

The result is 
TheOrem VI: Assuming that $X$ is smoothable, there are complexes

$$
\begin{aligned}
& H^{q-4}\left(X^{[k+2]}\right)(-2) \\
& \oplus \quad H^{q-2}\left(X^{[k+1]}\right)(-1) \\
& \longrightarrow H^{q-2}\left(X^{[k]}\right)(-1) \longrightarrow \oplus \longrightarrow H^{q}\left(X^{[k]}\right) \\
& \oplus \quad H^{q}\left(X^{[k-1]}\right) \\
& H^{q}\left(X^{[k-2]}\right)
\end{aligned}
$$

such that for $0 \leqq j \leqq m-i$

$$
H^{m-i}(-j) \cong H_{\text {Rest }}^{*} H_{\text {Gy }}^{*}\left(H^{m-i}\left(X^{[i+1]}\right)\right)(-j) .
$$

The notation in (VI.1) means that the left-hand side is computed by the cohomology of complexes in the statement of the theorem at the spot designated by the right-hand side in (VI.1). A significant fact here is that

$$
X \text { smoothable } \Longrightarrow \text { Rest } \circ \mathrm{Gy}=- \text { Gy } \circ \text { Rest . }
$$

The point is that this implication (VI.2) is generally not true unless $X$ is smoothable, although the result does not depend on any particular smoothing.

A further significant point is that in taking the cohomology of the restriction sequences

$$
\begin{aligned}
H_{\mathrm{Gy}}^{*}\left(H^{i}\left(X^{[j-1]}\right)(-m)\right) & \rightarrow H_{\mathrm{Gy}}^{*}\left(H^{i}\left(X^{[j]}\right)(-m)\right) \\
& \rightarrow H_{\mathrm{Gy}}^{*}\left(H^{i}\left(X^{[j+1]}\right)(-m)\right)
\end{aligned}
$$

we only put in $H^{i}\left(X^{[j]}\right)(-k)$ if $0 \leqq k<j-1$.

Referring to $(\underline{I .9})$ and Theorem $\nabla$ in the introduction, Theorem $\nabla \mathbf{D}$ implies that result, and the $N$-maps in the $N$-strings are the twisted identity maps on the individual pieces as given by (VI.1).

For the proof of Theorem VI one uses the basic constriction introduced in St1] and $[\mathrm{Zu}$. We will recall this for a standard family $X \stackrel{\pi}{\rightarrow} \Delta$ and observe at the end that the vector space $\mathbb{H}^{m}\left(\Omega_{X / \Delta}^{\bullet}(\log X) \otimes \mathcal{O}_{X}\right)$ has a filtration shifted down two steps by $N$, and the associated graded together with the mappings induced by $N$ may be defined in terms of $X$ alone. The $N$-strings that result are the ones expressed in the theorem. The upshot is that given an abstract normal crossing variety $X$ we will be able to define the object that is defined in terms of $X$ alone, provided only we assume that $X$ is smoothable. This object is related to the limiting mixed Hodge structure associated to the standard family in the manner just described. It is interesting to note that the object so described will be independent of the smoothing of $X$, provided that one exists. This is a reflection of the result in CKS1] that in a multi-parameter family the limiting mixed Hodge structure associated to the origin is independent of the direction of approach from the interior of the cone.

The construction in $[\mathrm{St1}]$ and $\mathrm{Zu}$ goes as follows: We define a bi-graded complex $A^{\bullet} \bullet$ where

$$
A^{p, q}=\Omega_{x}^{p+q+1}(\log X) / \widetilde{W}_{q} \Omega_{x}^{p+q+1}(\log X),
$$


where $\widetilde{W}_{q}$ is the standard filtration given by

$$
\widetilde{W}_{q}=\text { differential forms with at most } q d x_{i} / x_{i} \text { terms. }
$$

The differentials are given by

$$
\left\{\begin{array}{l}
d^{\prime}=\text { usual } d \\
d^{\prime \prime}=\wedge d t / t
\end{array}\right.
$$

The basic observation and definitions are

- The mapping $\Omega_{x}^{p}(\log X) \stackrel{\wedge d t / t}{\longrightarrow} A^{p, 0}$ has co-kernel naturally isomorphic to $\Omega_{x / \Delta}^{p}(\log X) \otimes \mathcal{O}_{X}$

- $W_{k} A^{p, q}=: \widetilde{W}_{2 q+k+1} \Omega_{x}^{p+q+1}(\log X) / \widetilde{W}_{q} \Omega_{x}^{p+q+1}(\log X)$;

- $F^{r} A^{p, q}= \begin{cases}A^{p, q} & \text { if } p \geqq r \\ 0 & \text { if } p<r\end{cases}$

Then with respect to $F^{\bullet}$ the map

$$
\Omega_{X / \Delta}^{\bullet}(\log X) \otimes \mathcal{O}_{X} \rightarrow A^{\bullet}
$$

is a filtered quasi-isomorphism. By definition $W \bullet A^{\bullet}$ induces the weight filtration on $\mathbb{H}^{m}\left(\Omega_{X / \Delta}^{\bullet}(\log X)\right) \otimes \mathcal{O}_{X}$. We note that

$$
\begin{aligned}
\operatorname{Gr}_{k}^{W} A^{p, q} & =W_{k+2 q+1} \Omega_{X}^{p+q+1}(\log X) / W_{k+2 q} \Omega_{X}^{p+q+1}(\log X) \\
& \cong \begin{cases}0 & \text { if } k \leqq-(q+1), \\
\Omega_{X^{[2 q+k+1]}}^{p-q-k} & \text { if } k \geqq-q\end{cases}
\end{aligned}
$$

where the second isomorphism is by the iterated residue map.

An intermediate step to computing $\mathbb{H}^{*}(A)$ is to use the spectral sequence associated to $\widetilde{W}$. For this spectral sequence where $A^{i}=\underset{p+q=i}{\oplus} A^{p, q}$ 


$$
\begin{aligned}
E_{1}^{a, b} & =H^{a+b}\left(\operatorname{Gr}_{-b}^{W} A^{i}\right) \\
& =H^{a+b}\left(\underset{q}{\oplus} \Omega_{X^{[2 q+1-b]}}^{i-2 q+b}\right) \\
& =\bigoplus_{q=\min (0, b)} H^{i-2 q+b, a+b}\left(X^{[2 q+1-b]}\right) .
\end{aligned}
$$

If $\operatorname{dim} X=n$, then $\operatorname{dim} X^{[2 q+1-b]}=n+1-(2 q+1-b)=n+b-2 q$, from which we have

$$
\begin{aligned}
H^{i-2 q+b, a+b}\left(X^{[2 q+1-b]}\right) \neq 0 \\
\Longrightarrow\left\{\begin{array}{l}
i-2 q+b \leqq n-2 q+b=\operatorname{dim} X^{[2 q+1-b]} \\
a+b \leqq n-2 q+b \Longleftrightarrow a \leqq n-2 q .
\end{array}\right.
\end{aligned}
$$

These are the only inequalities other than $\max (a, b) \leqq q \leqq i$. Thus to have potentially non-zero $H^{r, s}\left(X^{[t]}\right)$ we need

$$
\begin{aligned}
& i-2 q+b=r \\
& a+b=s \\
& t=2 q+1-b
\end{aligned} \Longleftrightarrow \begin{aligned}
& i=t+r+1 \\
& b=2 q+1-t \\
& a=s+t-2 q-1
\end{aligned} \quad(\Longrightarrow b \equiv 1-t(\bmod 2))
$$

and $q \geqq \max (0, b)$ gives $b \geqq \max (1-t, 2 b+1-t)$, which then gives

$$
\left\{\begin{aligned}
t-1 & \geqq b \geqq 1-t \\
b & \equiv 1-t(\bmod 2)
\end{aligned}\right.
$$

(thus $t=1, b=0 ; t=2, b=-1,1 ; t=3, b=-2,0,2, \ldots$ ) for $r=i-t+1=$ $i+\operatorname{dim} X^{[t]}-n=\operatorname{dim} X^{[t]}-(n-i)$. Fixing $t$ and noting $0 \leqq i \leqq n$ we then have

$$
H^{r, *}\left(X^{[t]}\right) \text { appears for one value of } i \text { and } t \text { values of } b \text {. }
$$

The above gives the conclusion that for $H^{n}=\mathbb{H}^{n}$ (LMHS), before cancellation in the spectral sequence

$$
\begin{array}{cll}
H^{n}\left(X^{[1]}\right) & \text { appears once } & (b=0) \\
H^{n-1}\left(X^{[2]}\right) & \text { appears once } & (b=-1,1) \\
H^{n-2}\left(X^{[3]}\right) & \text { appears three times } & (b=-2,0,2) \\
\vdots &
\end{array}
$$

Sketch of the proof of Theorem IV] Referring now to (VI.3), by [St1 the spectral sequence degenerates at $E_{2}$. The $E_{1}$-terms are as indicated there. The $d_{1}$-map is, as noted in $[\mathrm{Zu}$, "composed of various restriction and Gysin maps." After unwinding the indices, the $d_{1}$-complex turns out to be the one in the statement of the theorem.

We now come to the main point. Note that the individual terms and maps may be defined in terms of $X$ alone. However in general we do not have

$$
\text { Rest } \circ \text { Gy }=- \text { Gy } \circ \text { Rest . }
$$

Proposition: The anti-commutativity commutativity (VI.4) holds if, and only if, $\mathcal{O}_{D}(X)$ is topologically trivial. This is the case if $X$ is smoothable. 
Rather than give the formal argument we shall indicate by example in the simplest non-trivial cases why the result should be true 31 We note that if $X$ is smoothable the proposition is true. Our central point is that this sufficient condition is essentially also necessary. We say essentially, because (VI.4) is a purely topological fact which only requires that $\mathcal{O}_{D}(X)$ be topologically, but not necessarily analytically, trivial.

Let $X$ be an irreducible surface having as singular locus a double curve $C$ whose inverse image in $X^{[1]}$ is a disjoint union $X^{[1]}=C_{1} \amalg C_{2}$ of two smooth curves. We will denote by $H^{q}\left(X^{[2]}\right)_{\text {- the classes }} \alpha \oplus-\alpha \in H^{q}\left(C_{1} \amalg C_{2}\right)$. Then $H^{q}\left(X^{[2]}\right)_{-} \cong$ $H^{q}(C)$, but we put opposite signs on those in $H^{q}\left(C_{1}\right)$ and $H^{q}\left(C_{2}\right)$. Then we shall show

(i) $\mathcal{O}_{D}(X)$ is topologically trivial if, and only if, $C_{1}^{2}=-C_{2}^{2}$;

(ii) the sequence

$$
H^{0}\left(X^{[2]}\right)_{-}(-1) \stackrel{\text { Gy }}{\longrightarrow} H^{2}\left(X^{[1]}\right) \stackrel{\text { Rest }}{\longrightarrow} H^{2}\left(X^{[2]}\right)_{-}
$$

is a complex if, and only if, $C_{1}^{2}=-C_{2}^{2}$.

The complex (VI.5) is the simplest non-trivial case of the sequences that appear in the statement of Theorem VI.

Denoting by $\eta_{C_{i}} \in H^{2}\left(X^{[1]}\right)$ the fundamental class of $C_{i}$ and by $\left[C_{i}\right]$ the fundamental class of $C_{i}$ itself, the sequence (VI.5) is

$$
\begin{gathered}
1_{C_{1}}-1_{C_{2}} \rightarrow \eta_{C_{1}}-\eta_{C_{2}} \longrightarrow \quad\left\{\left(\left(C_{1}-C_{2}\right) \cdot C_{1}\right)\left[C_{1}\right]+\left(\left(C_{1}-C_{2}\right) \cdot C_{2}\left[C_{2}\right]\right)\right\} \\
\| \\
\left\{C_{1}^{2}\left[C_{1}\right]-C_{2}^{2}\left[C_{2}\right]\right\}_{-} \\
\| \\
\left(\frac{C_{1}^{2}+C_{2}^{2}}{2}\right)\left(\left[C_{1}\right]-\left[C_{2}\right]\right),
\end{gathered}
$$

which proves (ii).

As for (i) we have

$$
N_{D}(X) \cong N_{C_{1}}\left(X^{[1]}\right) \otimes N_{C_{2}}\left(X^{[1]}\right)^{*}
$$

In case $X$ is still an irreducible surface a piece of the complex in Theorem VI is

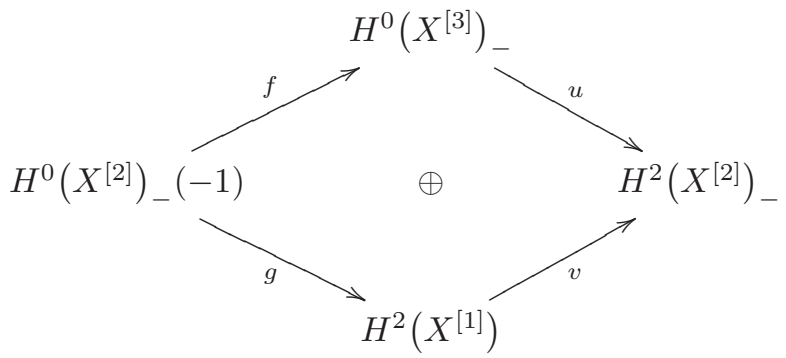

Here the minus sign on ( ) _ refers to classes that transform by the sign of the induced action on cohomology given the labeling into even-odd of the components lying over a general point in the map $X^{[k]} \rightarrow X_{k}$. The conditions to have a complex are

$$
u \cdot f+v \cdot g=0
$$

which when worked out is a consequence of $\mathcal{O}_{D}(X) \cong \mathcal{O}_{D}$.

\footnotetext{
${ }^{31}$ The details of this argument will appear in the previously mentioned work in progress.
} 


\section{REFERENCES}

$\left[\mathrm{A}^{\dagger}\right]$ D. Abramovich, Q. Chen, D. Gillam, Y. Huang, M. Olsson, M. Satriano, and S. Sun, Logarithmic geometry and moduli, available at http://www.math.brown.edu/ abromovic/LOGGEOM/master.pdf.

[AK] D. Abramovich and K. Karu, Weak semistable reduction in characteristic 0, Invent. Math 139 (2000), 241-273.

[AMRT] A. Ash, D. Mumford, M. Rapoport, and Y. Tai, Smooth compactification of locally symmetric varieties, in Lie Groups: History, Frontiers and Applications, Vol. IV, Math. Sci. Press, Brookline, MA, 1975.

[Ca] J. A. Carlson, The geometry of the extension class of a mixed Hodge structure, Proc. Sympos. Pure Math. 46 (1987), 199-222.

[Ca-Fe] J. Fernandez and E. Cattani, Infinitesimal variations of Hodge structure at infinity, Geom. Dedicata 139 (2009), 299-312.

[CK] E. Cattani and A. Kaplan, Polarized mixed Hodge structures and the local monodromy of a variation of Hodge structure, Invent. Math. 67 (1982), 101-115.

[CKS1] E. Cattani, A. Kaplan, and W. Schmid, Degenerations of Hodge structures, Ann. of Math. 123 (1986), 457-535.

[CKS2],$L^{2}$ and intersection cohomologies for a polarizable variation of Hodge structure, Invent. Math. 87 (1987), 217-252.

[De] P. Deligne, Équations Differentielles à Points Singulars Réguliere, Springer Lect. Notes, Springer-Verlag, New York, 163, 1970.

[Fr1] R. Friedman, The period map at the boundary of muduli, in Topics in Transcendental Algebraic Geometry, 183-208, (P. Griffiths, ed.), Princeton Univ. Press, Princeton, NJ, 1984.

[Fr2] Global smoothings of varieties with normal crossings, Ann. of Math. 118 (1983), $75-114$.

[Fu1] T. Fujisawa, Limits of Hodge structures in several variables, Compos. Math. 115 (1999), $129-183$.

[Fu2] Mixed Hodge structures on log smooth degenerations, Tohoku Math. 60 (2008), $71-100$.

[FHW] G. Fels, A. Huckelberry, and J. A. Wolf, Cycle Spaces of Flag Domains, A Complex Geometric Viewpoint, Progr. Math. 245, (H. Bass, J. Oesterlé, and A. Weinstein, eds.), Birkhäuser, Boston, 2006.

[GG] M. Green and P. Griffiths, Reduced limit period mappings and orbits in Mumford-Tate varieties, to appear.

[GGK] M. Green, P. Griffiths, and M. Kerr, Hodge Theory, Complex Geometry and Representation Theory, CBMS 118, Amer. Math. Soc., Providence, RI, 2013.

[GGR] M. Green, P. Griffiths, and C. Robles, Extremal degenerations of polarized Hodge structures, to appear.

[KN] Y. Kawawmata and Y. Namikawa, Logarithmic deformations of normal crossing varieties smoothen of degenerate Calabi Yau varieties, Invent. Math. 118 (1994), 395-409.

[KP1] M. Kerr and G. Pearlstein, Boundary components of Mumford-Tate domains. arXiv:1210.5301

[KP2] Naïve boundary strata and nilpotent orbits, preprint, 2013.

[KU] K. Kato and S. Usui, Classifying Spaces of Degenerating Polarized Hodge Structures, Ann. of Math. Stud. 169, Princeton Univ. Press, Princeton, NJ, 2009.

[Pa] V. P. Palamodov, Deformations of complex spaces, Russian Math. Surveys 31, (1976), 128-197; translated from Uspekhi Mat. Nauk. 31 (1976), 129-194.

[PS] C. A. M. Peters and J. H. M. Steenbrink, Mixed Hodge Structures, Ergeb. Math. Grenzgeb. 52, Springer-Verlag, New York, 2008.

[Ro] C. Robles, working notes at the IAS.

[Sc] W. Schmid, Variation of Hodge structure: the singularities of the period mapping, Inv. Math. 22 (1973), 211-319.

[St1] J. Steenbrink, Limits of Hodge structures, Invent. Math. 31 (1969), 229-257.

[St2] Logarithmic embeddings of varieties with normal crossings and mixed Hodge structures, Math. Ann. 301 (1995), 105-118. 
[St-Zu] J. Steenbrink and S. Zucker, Variation of mixed Hodge structure. I, Invent. Math. 80 (1985), 489-542.

[Zu] S. Zucker, Degeneration of Hodge bundles (after Steenbrink), in Topics in Transcendental Algebraic Geometry, 121-142, (P. Griffiths, ed.), Princeton Univ. Press, Princeton, NJ, 1984.

Department of Mathematics, University of California at

Los Angeles, Los Angeles, CA 90095

E-mail address: mlg@ipam.ucla.edu

Institute for Advanced Study, Einstein Drive, Princeton, NJ 08540

E-mail address: pg@ias.edu 